\title{
Frame effects in visual search for line orientation
}

\author{
SHUJI MORI \\ Tokyo Metropolitan University, Tokyo, Japan \\ and \\ AKIHITO KATAOKA \\ Hitachi Software Engineering Co., Ltd., Tokyo, Japan
}

\begin{abstract}
In the present study, we investigated the effects of surrounding frames on visual search for line orientation. Every line item presented in the display was surrounded by a square frame of identical size and orientation. The orientations of the frames, as well as those of the target and distractor lines, were either vertical or tilted. In six experiments, the surrounding frames caused substantial changes in search efficiency for vertical targets and for tilted targets. The search asymmetry between the two types of targets was reversed when the frame was tilted at the same angle as the tilted line. Several variations of the frames (a pair of parallel lines, squares with gaps, and squares with circular contours inside) also changed search efficiency significantly. Taken together, these results imply that three different sources contribute to frame effects: distractor roles played by the frame components, orientation contrast from the frame contour, and interference in local orientation processing (Hayward \& Burke, 2000). Implications of the present findings are discussed in reference to rod-and-frame effects (Witkin $\&$ Asch, 1948) and to the effects of a large frame surrounding an entire display of lines (Marendaz, 1998; Treisman, 1985).
\end{abstract}

Visual context serves as a frame of reference for the apparent orientation of an object. A well-known example is rod-and-frame (RF) effects. As Witkin and Asch (1948) first demonstrated, observers make more errors in setting a rod to a perceptually vertical position when the rod is placed inside a tilted frame than when it is presented with a gravitationally vertical frame or with no frame. A usual interpretation of the RF effect is that, in addition to gravity, the tilted frame serves as a frame of reference for perception of the upright (Rock, 1990). The observers perceive rod orientation in reference to frame orientation and to gravity, and the resulting rod setting is a compromise between the two references. Subsequent studies have shown that RF effects vary with a number of variables, such as frame size (Ebenholtz, 1977; Spinelli, An-

Experiments 1, 3, 4, and 5 were conducted by the second author as part of a master's thesis submitted to Toyama Prefectural University. The data have been previously reported in a somewhat different form at the 40th Annual Meeting of the Psychonomic Society, Los Angeles, November 1999. The research was supported by Grants-in-Aids for Scientific Research 09710054, 11610083, and 13610095 to S.M. and 14101001 to Yoshitaka Nakajima. We thank Cecile Ballaz and Ann Treisman for providing information regarding their experiments and Takashi Kusano and Takayuki Ishiwata for assistance in programming and manuscript preparation. We also thank Jeremy Wolfe, Joshua Solomon, and Christian Marendaz for valuable suggestions. Correspondence concerning this article should be addressed to S. Mori, Faculty of Science, Department of Kinesiology, Tokyo Metropolitan University, 1-1 Minami-Ohsawa, Hachioji, Tokyo 192-0397, Japan (e-mail: smori@comp.metro-u.ac.jp).

Note-This article was accepted by the previous editorial team, headed by Neil Macmillan. tonucci, Goodenough, Pizzamiglio, \& Zoccolotti, 1991), frame tilt (Beh, Wenderoth, \& Purcell, 1971), the distance between the rod and the frame (Coren \& Hoy, 1986; Zoccolotti, Antonucci, \& Spinelli, 1993), and the observer's posture (Zoccolotti, Antonucci, Goodenough, Pizzamiglio, \& Spinelli, 1992).

The effects of a tilted frame are not limited to setting a rod. Using a visual search task, Treisman (1985) demonstrated that search performance for a unique line orientation depended on the orientation of a frame surrounding an entire display of lines. The orientation of the lines was either gravitationally vertical or tilted $18^{\circ}$ from the verti$\mathrm{cal}$, and the observer's task was to search for a vertical line among tilted lines or to search for a tilted line among vertical lines. A main dependent variable of the observer's performance was search rate, which was represented by the slope of a linear function relating reaction times (RTs) to the number of items in the display. In the absence of a surrounding frame, search for a tilted line was fast, with a rate less than $5 \mathrm{msec} /$ item, whereas search for a vertical line was much slower, the rate being $28 \mathrm{msec} /$ item. A similar asymmetry for search performance between tilted and vertical targets was observed when the lines were presented inside a vertical rectangular frame. When the frame was tilted $18^{\circ}$ from the vertical, the same angle as the tilted line, search performance was quite different; search for a vertical line became faster than search for a tilted line, with rates of 3 and $10 \mathrm{msec} /$ item, respectively. It seems that the asymmetry between the two search tasks was reversed by the tilted frame.

In a similar experiment, Marendaz (1998) used vertical and $18^{\circ}$-tilted lines as stimuli, with the frame vertical 
or tilted $18^{\circ}$ or $36^{\circ}$ from the vertical. When the frame was vertical, an asymmetry of search performance was observed, with rates of approximately $5 \mathrm{msec} / \mathrm{item}$ for a tilted target and $40 \mathrm{msec} / \mathrm{item}$ for a vertical target. When the frame was tilted $18^{\circ}$, the asymmetry disappeared, with rates of about $10 \mathrm{msec} /$ item for both search tasks. When the frame was further tilted $36^{\circ}$, the asymmetry reappeared in a manner similar to that with the vertical frame. These results suggest that a tilted frame is most effective when visual search involves vertical lines and lines tilted at the same angle as the frame.

Like RF effects, the effects of a tilted frame on visual search performance have been interpreted in terms of a reference system for the coding of line orientation (Marendaz, 1998; Treisman, 1985). Treisman (1985; Treisman \& Gormican, 1988) has proposed that line orientation is coded in reference to a standard point on the orientation dimension: Lines are positively coded when their orientations deviate from the standard, whereas lines at the standard orientation are coded as neutral. In the visual search task, a target with a positively coded orientation is easily and rapidly detected among distractors with a standard orientation, which signal only a null value. In the absence of a surrounding frame, the standard is the vertical, defined by gravity and the retinal coordinate system. Search for a tilted line among vertical lines is fast because the tilted line is the only item in the display that is positively coded among the null values of the orientations of the vertical lines. On the other hand, search for a vertical line among tilted lines is slow because the null value of the target is masked by signals of positive values for the distractors. When the lines are surrounded by a frame, the standard orientation shifts to the frame orientation. For a vertical frame, the standard orientation remains the gravitational vertical, and the search asymmetry between a tilted and a vertical target is unchanged. For a frame tilted $18^{\circ}$, the standard orientation shifts to $18^{\circ}$, and the vertical lines are more positively coded than are the tilted lines. This explains the reversed asymmetry of search performance.

Marendaz (1998; Marendaz, Stivalet, Barraclough, \& Walkowiac, 1993) has offered an explanation based on Foster and Ward's (1991a, 1991b) model of orientationselective filters. According to the model, search performance for lines with different orientations is determined by the outputs of two orthogonal filters, one tuned to around $0^{\circ}$ and the other to around $90^{\circ}$, which are activated by target and distractor lines. In the visual search task involving $18^{\circ}$-tilted and vertical $\left(0^{\circ}\right)$ lines, the tilted line activates the two filters, but the vertical line activates only the $0^{\circ}$ filter, resulting in faster detection of a tilted target than of a vertical target. This explains the search asymmetry observed with no surrounding frame. Extending this model, Marendaz hypothesized that the tuning functions of the two filters are dynamically determined in reference to frame orientation, as well as to gravity information (but see Doherty \& Foster, 2001). He suggested that a tilted frame may be involved in sur- face representation, which is thought to precede and affect visual search processes (e.g., He \& Nakayama, 1992).

In either explanation, the surrounding frame plays a key role in visual search for line orientation. In the present study, we conducted a series of visual search experiments using a different type of frame, which we refer to as the local frame (LF), to differentiate it from the type of frame used by Treisman (1985) and Marendaz, which we refer to as the global frame (GF). Examples of LFs are shown in Figure 1. For the LF, each line in the display was surrounded by a single frame, in a way similar to the configuration typically used in RF studies. As was discussed above, the work of Treisman (1985) and Marendaz has much in common with the research on RF effects: Effects of the tilted frame on the perception of the orientation of line segments inside the frame are measured, and the effects are interpreted as indicating that frame orientation served as a frame of reference for line orientation. In fact, Marendaz noted that his results for the effects of a frame on visual search resembled those for the RF effects reported by Spinelli et al. (1991). One major difference between the two types of experiments is that a single line segment (rod) is presented inside a frame in RF experiments, whereas multiple lines are presented with a single surrounding frame in visual search experiments. In RF experiments, an observer's error in rod setting is attributable, with little ambiguity, to the apparent orientation of the rod, and the observed RF effects can be specified by the physical properties of the rod and the frame, such as their size and orientation and the physical distance between them (e.g., Spinelli et al., 1991; Wenderoth, 1974; Zoccolotti et al., 1993). On the other hand, it is not very clear from visual search experiments whether the surrounding frame affects the apparent orientation of a target line, distractor lines, or both. Since these lines are distributed randomly in a search display inside the frame, the physical distance between the lines and the frame is not controlled. In the present study, we introduced the LF in order to relate frame effects in visual search tasks more closely to RF effects. As compared with those for large surrounding frames, the results for an LF would be more easily related to RF effects in relevant conditions. In some of the present experiments, we manipulated the physical properties of the LF, as has been done in studies of RF effects (Streibel, Barnes, Julness, \& Ebenholtz, 1980; Wenderoth \& Beh, 1977).

\section{EXPERIMENT 1}

This experiment provided the basis for the subsequent experiments in the present study. We examined the effects of LFs, vertical or tilted, in search tasks for a vertical line and for a tilted line. We also examined the effects of GFs for comparison.

\section{Method}

Subjects. Twelve undergraduate students at Toyama Prefectural University (all males, 19-21 years of age) participated in this ex- 
(A)

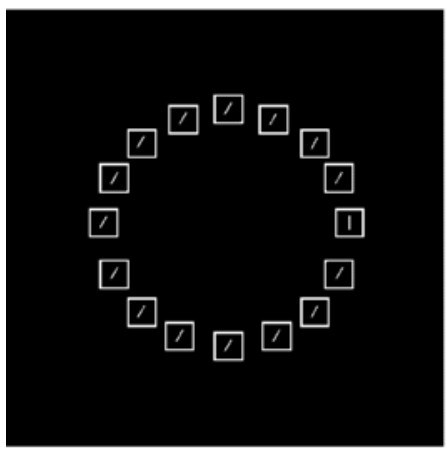

(C)

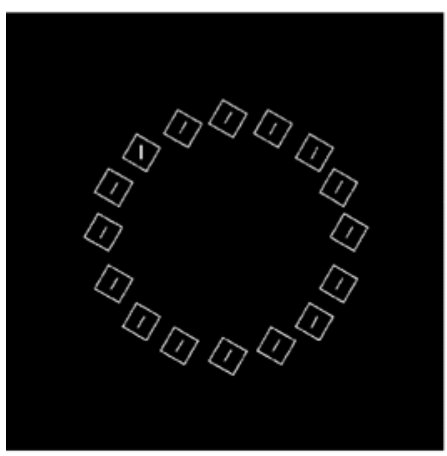

(E)

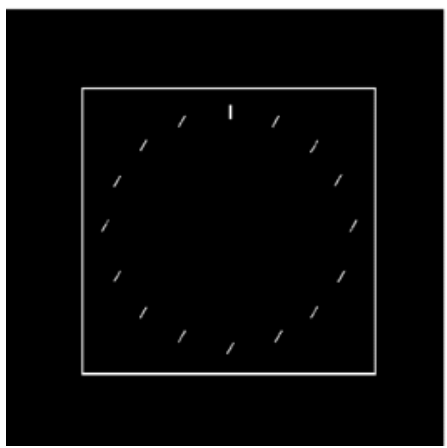

(B)

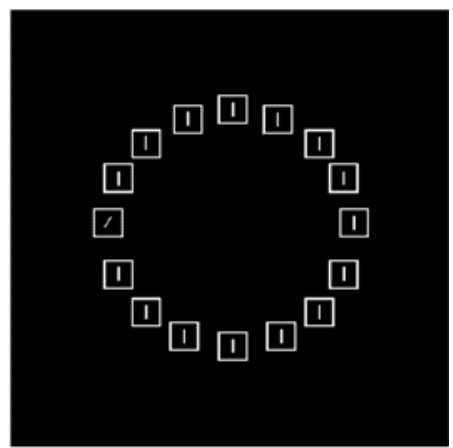

(D)

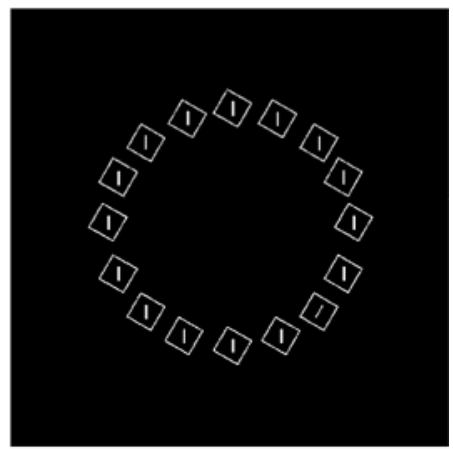

(F)

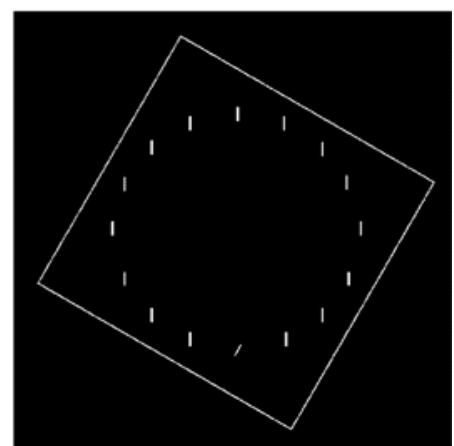

Figure 1. Examples of search displays in Experiment 1. All the examples represent 16-item target-present trials, the left ones showing the search for a vertical target and the right ones for a tilted target. Panels $A$ and $B$ show vertical local frames (LFs), panels $C$ and D show tilted LFs, and panels $E$ and $F$ show vertical and tilted global frames (GFs), respectively.

periment for course credit. All had normal or corrected-to-normal vision, and none claimed to have difficulty seeing the items presented in the display. All the subjects were naive as to the purpose of the present study.

Apparatus and Stimuli. The stimuli were presented on an Apple Multiple Scan 17-in. monitor controlled by a Power Macintosh $8100 / 1000 \mathrm{AV}$ computer using Vscope software. The screen area was $24 \times 32 \mathrm{~cm}$ (or a visual angle of $19.7^{\circ} \times 26.4^{\circ}$ ) in height and width, and other parts of the monitor were masked by a rectangular black board (which was visible during the experiment). The screen was viewed binocularly from an approximately $70-\mathrm{cm}$ dis- tance under dim illumination. The stimuli were presented in white $\left(72.0 \mathrm{~cd} / \mathrm{m}^{2}\right)$ on a black background (no pixel lit). Target and distractor items were line segments of visual angle $0.6^{\circ}$ in length, either vertical or tilted $30^{\circ}$ right from the vertical. ${ }^{1}$ Note that the angular difference used in the present study was larger than the $18^{\circ}$ difference typically used in previous studies (Marendaz et al., 1993; Treisman, 1985).

As is shown in Figure 1, items were distributed randomly at 16 possible positions evenly located on an imaginary circle approximately $10^{\circ}$ in diameter (no jitter was added to any of the item positions). The circular presentation was used to control the eccentric- 
ity of all the items from a fixation point centered at the display, since search performance varies considerably with item fixation eccentricity (e.g., Carrasco, McLean, Katz, \& Frieder, 1998; Wolfe, O'Neill, \& Bennett, 1998). In the LF condition, each line in the display was surrounded by a frame subtending $1.3^{\circ}$ in height and width (Figures 1A-1D). In the GF condition, all the items were surrounded by a square frame subtending approximately $12.3^{\circ}$ in height and width (Figures $1 \mathrm{E}$ and $1 \mathrm{~F}$ ). Both the LF and the GF were either vertical or tilted $30^{\circ}$ right. Note that in the LF condition, all the frames were of the same orientation (vertical or tilted). In the no-frame (NF) condition, the line items were presented alone.

Procedure. The subjects were seated on a chair with the head upright. No apparatus was used to support the head position, and the subjects maintained their posture and head position by themselves during the experimental sessions. At the beginning of each trial, a white dot, serving as a fixation point, was presented at the center of the screen. After $670 \mathrm{msec}$, the white dot was replaced by the stimulus display, which remained visible until the subject responded. The subjects were instructed to respond as rapidly as possible while keeping errors below $10 \%$. They responded by pressing one of two response keys on a computer keyboard, using the index and middle fingers of their right hands for target-present and targetabsent responses, respectively. Accuracy feedback (plus or minus sign) was displayed at the center of the screen after each response. The intertrial interval was $840 \mathrm{msec}$.

The task was either to search for a tilted line among vertical lines, or to search for a vertical line among tilted lines. The two tasks were assigned to separate groups of 6 subjects each, and each subject performed the task for the five conditions: NF, GF vertical, GF tilted, LF vertical, and LF tilted. The order of conditions (one session for each condition) was randomized across the subjects in each group. One session consisted of one practice block and three experimental blocks of 64 trials each. A target was presented in a random half of the total trials in one session. Set sizes of $1,4,10$, and 16 items were used. Set size, position of target and distractors, and target presence and absence were randomized across trials.

\section{Results}

Figures 2 and 3 show the mean correct RTs for targetpresent and target-absent trials, respectively, in the search tasks for a vertical target and for a tilted target $(6$ subjects each) with the three frame types. The search rates for the RT data, computed by a linear regression of the mean RTs on set size, are also shown in the figures. As can be readily seen from the figures, search performance varied considerably by frame type and orientation, target orientation, and target presence or absence. In the NF condition, there was an asymmetry between search for a tilted target and search for a vertical target. The mean RTs were entered into a 2 (target type: vertical or tilted) $\times 2$ (trial type: target presence or target absence) $\times 4$ (set size: $1,4,10$, or 16) analysis of variance (ANOVA) ${ }^{2}$ which showed significant main effects of target type $[F(1,10)=6.27, p<.05]$, trial type $[F(1,10)=$ $8.77, p<.05]$, and set size $[F(3,30)=35.88, p<.01]$ and a significant interaction of target type and set size $[F(3,30)=9.29, p<.01]$.

In the LF condition, a large asymmetry between the two search tasks was observed for both frame orientations. More important, the asymmetry for the tilted LF (open and filled squares) was reversed from that for the vertical LF (open and filled diamonds). A four-factor (target type, frame orientation, trial type, and set size)
ANOVA showed significant main effects of frame orientation $[F(1,10)=35.43, p<.01]$, trial type $[F(1,10)=$ $66.15, p<.01]$, and set size $[F(3,30)=229.15, p<.01]$ and significant interactions of target type and frame orientation $[F(1,10)=74.32, p<.01]$, frame orientation and set size $[F(3,30)=12.65, p<.01]$, trial type and set size $[F(3,30)=18.12, p<.05]$, and target type, frame orientation, and set size $[F(3,30)=79.89, p<.01]$. There was no effect of target type $[F(1,10)=0.24]$, which seems to reflect the opposing patterns of asymmetry produced by the two frame orientations, which canceled out each other's effects. This inference was confirmed by separate ANOVAs for the two frame orientations. For the vertical LF, there were significant main effects of target type $[F(1,10)=5.84, p<.05]$, trial type $[F(1,10)=41.22, p<.01]$, and set size $[F(3,30)=102.01, p<.01]$ and significant interactions of target type and set size $[F(3,30)=19.49, p<.01]$ and trial type and set size $[F(3,30)=13.04, p<.01]$. For the tilted LF, the same main effects and interactions were significant [target type, $F(1,10)=15.43, p<.01$; trial type, $F(1,10)=44.24, p<.01$; set size, $F(3,30)=$ $232.07, p<.01$; target type $\times$ set size, $F(3,30)=32.86$, $p<.01$; trial type $\times$ set size, $F(3,30)=8.02, p<.01]$. In comparison with the NF condition, both frame orientations produced slower searches for the two types of targets. For the vertical LF, this was confirmed by ANOVAs showing that the difference in RTs between the vertical LF and the NF conditions interacted significantly with set size for a vertical target $[F(3,15)=17.01]$ and for a tilted target $[F(3,15)=14.47$; both $p$ s $<.01]$. Analyses for the tilted LF showed similar results.

In the GF condition, search efficiency in the two search tasks also varied with frame orientation. For the vertical GF (squares), the asymmetry between the two search tasks was similar to that observed in the NF condition. For the tilted GF (diamonds), the asymmetry appeared to be shrunken, particularly in the absent trials. A four-factor ANOVA showed a significant main effect of set size $[F(3,30)=47.90, p<.01]$, but none of the other main effects (target type, frame orientation, and trial type) was significant. There were significant interactions of target type and set size $[F(3,30)=14.50, p<$ $.01]$, trial type and set size $[F(3,30)=2.93, p<.05]$, target type, frame orientation, and trial type $[F(1,10)=$ $5.94, p<.05]$, and target type, frame orientation, and set size $[F(3,30)=5.77, p<.05]$. For the vertical GF, an ANOVA showed significant main effects of target type $[F(1,10)=8.52, p<.05]$ and set size $[F(3,30)=34.33$, $p<.01]$ and a significant interaction of target type and set size $[F(3,30)=22.26, p<.01]$, proving the asymmetry between the two search tasks. For the tilted GF, only a main effect of set size was significant $[F(3,30)=$ $20.32, p<.01]$. The lack of a main effect of target type $[F(1,10)=1.20]$ and an interaction of target type and set size $[F(3,30)=2.78]$ confirmed that the search asymmetry was much reduced by the tilted GF. When the data for the NF condition were added to an ANOVA, there 

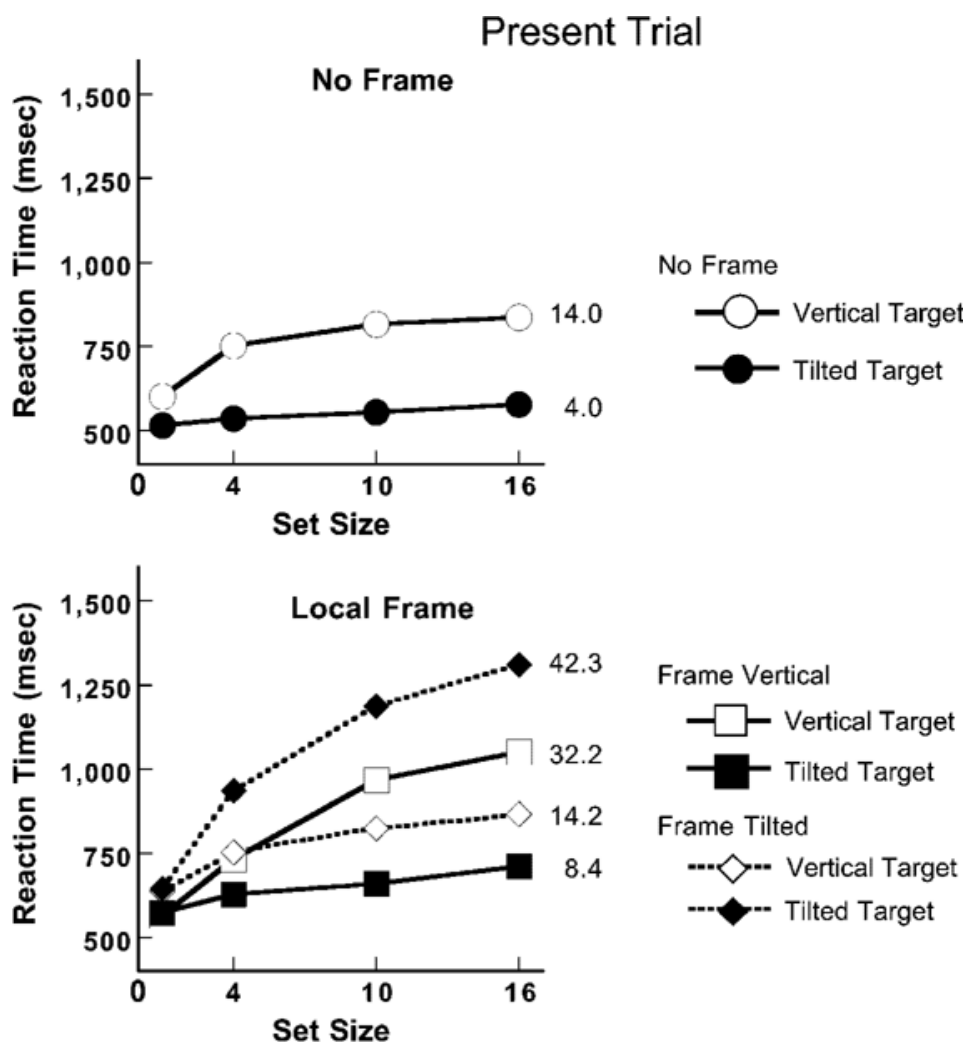

Frame Tilted

-.>- Vertical Target

--- Tilted Target

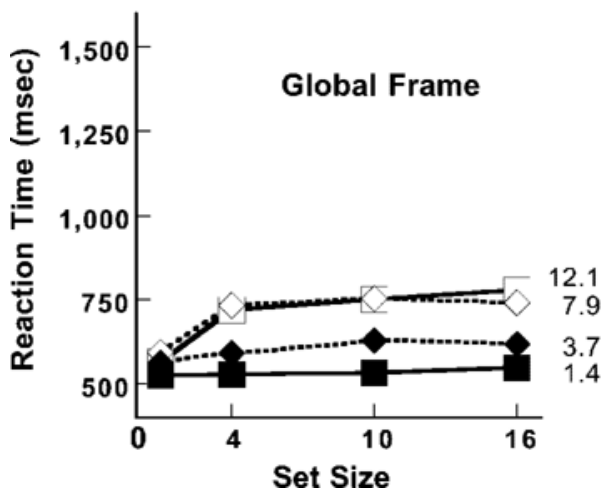

Figure 2. Mean correct reaction times (RTs) in the present trials in Experiment 1 . The number at the right of each $R T$ function is the slope, or search rate (msec/item), of a linear regression fitted to the function.

was no significant difference among the NF condition and the two frame orientations $[F(2,20)=1.79]$.

Table 1 presents the percentages of errors in Experiment 1 . The errors were higher in the present trials than in the absent trials, as has often been observed in other visual search experiments (e.g., Mori, 1997). Since RTs were shorter in the present trials (Figures 2 and 3), the differences in RTs between the present and the absent trials may have been contaminated by a speed-accuracy tradeoff. In general, errors increased with increases in set size for the conditions that produced slow search rates (e.g., a vertical target with the LF vertical, a tilted target with the LF tilted). To verify this observation, we computed the correlations between the mean RTs and the percentages of errors separately for the present and the absent trials. A negative correlation, if significant, would indicate possible contamination by a speed-accuracy tradeoff in the observed effects on RTs. The correlations were positive in the present and the absent trials $[R(38)=.883$ and .522 , respectively; both $p \mathrm{~s}<.01]$. For the vertical target alone, $R(18)=.897$ in the present trials, and $R(18)=.641$ in the absent trials (both $p \mathrm{~s}<.01$ ). For the tilted target alone, $R(18)=.906, p<.01$, in the present trials, and $R(18)=$ .442 , n.s., in the absent trials. Therefore, it is safe to say 

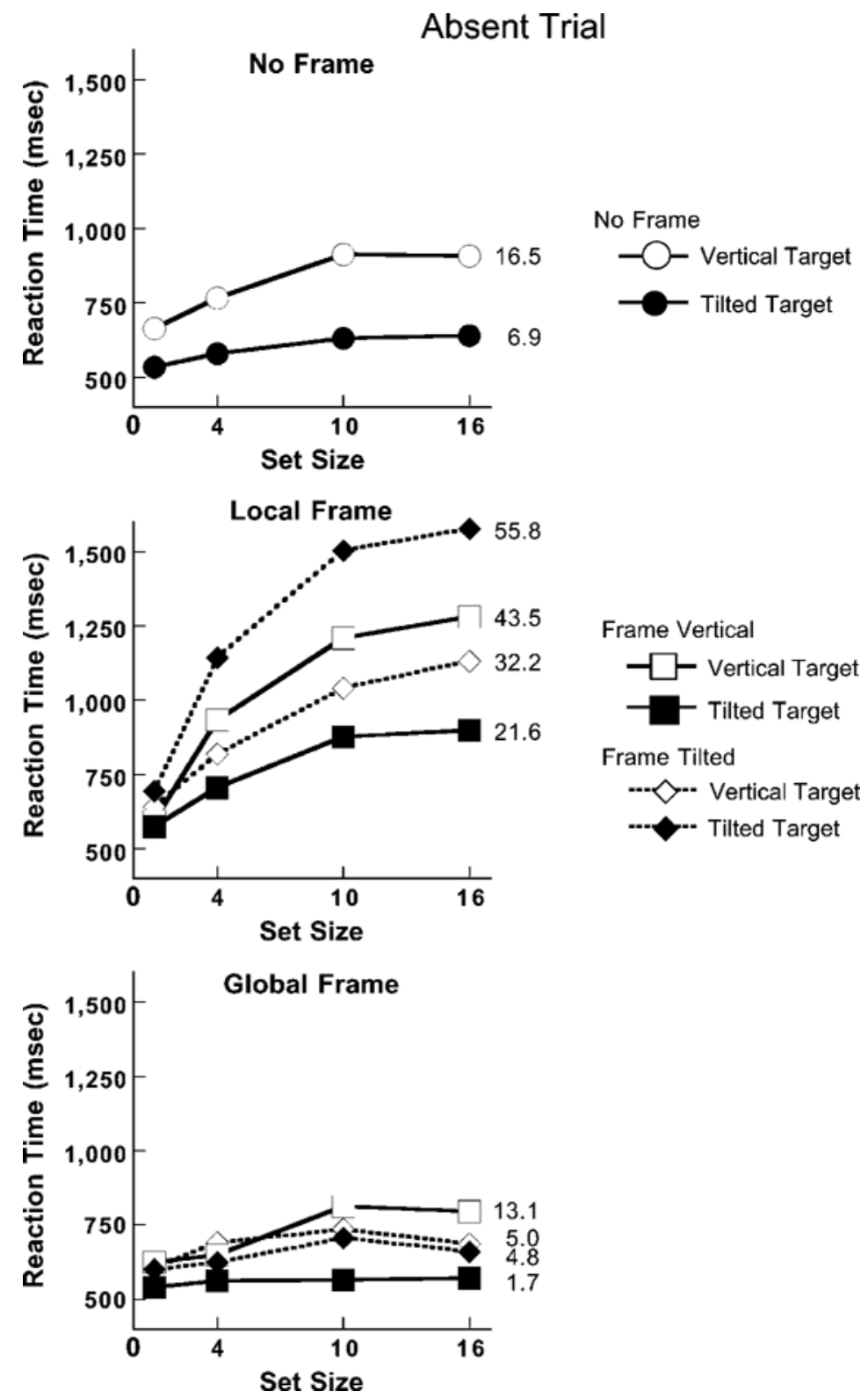

Figure 3. Mean correct reaction times (RTs) in the absent trials in Experiment 1 , together with the search rates (msec/item) for the RT functions.

that the differences in RTs among the frame types and target orientations did not reflect a speed-accuracy tradeoff.

\section{Discussion}

The reversal of search asymmetry by the tilted LF is similar to the finding of Treisman (1985) for a large surrounding frame and is in good agreement with her account of the frame effect. In line with her account, we hypothesize that line orientation would be coded in relation to the standard set by the orientation of the LF. For the vertical LF, the standard would be vertical, and the tilted lines would be positively coded, whereas the verti- cal lines would signal only null values. This explains the faster search for the tilted line than for the vertical line. For the tilted LF, the standard would shift to the frame orientation $\left(30^{\circ}\right.$ right $)$, and the lines tilted at the same angle as the frame would be coded as neutral, which would lead to a slower search for the tilted line than for the vertical line - that is, a reversed asymmetry.

Although the reference standard hypothesis fits the data quite well, other explanations are also possible. One strong possibility is that target detection was distracted by the frame contour. In the search for a tilted line, the target line was of the same orientation as the left and 
Table 1

Percentages of Errors in Experiment 1

\begin{tabular}{|c|c|c|c|c|c|c|c|c|c|}
\hline \multirow[b]{2}{*}{ Frame } & \multirow[b]{2}{*}{ Target } & \multicolumn{4}{|c|}{ Present Trial } & \multicolumn{4}{|c|}{ Absent Trial } \\
\hline & & 1 & 4 & 10 & 16 & 1 & 4 & 10 & 16 \\
\hline \multirow[t]{2}{*}{$\mathrm{NF}$} & Vertical & 1.4 & 2.8 & 7.6 & 11.8 & 4.9 & 2.8 & 2.1 & 2.1 \\
\hline & Tilted & 2.1 & 0.0 & 2.8 & 4.2 & 2.1 & 2.1 & 0.0 & 0.7 \\
\hline \multirow[t]{2}{*}{ GF vertical } & Vertical & 4.2 & 3.5 & 7.6 & 8.3 & 0.7 & 2.1 & 3.5 & 1.4 \\
\hline & Tilted & 5.6 & 2.1 & 4.2 & 5.6 & 4.2 & 2.8 & 0.7 & 0.0 \\
\hline \multirow[t]{2}{*}{ GF tilted } & Vertical & 2.1 & 5.6 & 8.3 & 6.9 & 2.1 & 0.0 & 1.4 & 2.8 \\
\hline & Tilted & 2.1 & 2.1 & 1.4 & 9.0 & 4.9 & 2.1 & 2.1 & 0.7 \\
\hline \multirow[t]{2}{*}{ LF vertical } & Vertical & 0.7 & 4.9 & 20.1 & 19.4 & 3.5 & 4.9 & 4.9 & 7.6 \\
\hline & Tilted & 4.2 & 4.9 & 5.6 & 8.3 & 1.4 & 1.4 & 2.8 & 0.0 \\
\hline \multirow[t]{2}{*}{ LF tilted } & Vertical & 4.9 & 2.8 & 9.0 & 10.4 & 2.8 & 1.4 & 3.5 & 4.2 \\
\hline & Tilted & 4.2 & 9.0 & 15.3 & 29.2 & 2.8 & 3.5 & 4.2 & 4.2 \\
\hline
\end{tabular}

Note-NF, no frame; GF, global frame; LF, local frame.

right component lines of the tilted LF (see Figure 1D). The color and the width of the component lines were also the same as those of the target line, and their only difference was in length. Therefore, the two component lines might have acted as additional distractors, and the observers might have searched for a tilted line among the lines of the same length but a different orientation (vertical distractors) and those of the same orientation but a different length (component lines). Searches for a conjunction of two different features are generally slow, as compared with searches for a single feature (Treisman, 1988; Treisman \& Sato, 1990; but see Wolfe, 1999). When the target was a vertical line, the target line and the left or right component line were different in orientation (see Figure 1C), and the observers could simply search for the target among the vertical lines (distractors and component lines). As a result, search for a vertical target would have become faster than search for a tilted target in the tilted LF condition. The same argument applies to the vertical LF condition. Here, the vertical target would have been distracted by the component lines, because they were of the same orientation as the target (Figure 1A). This might have contributed to the relatively slow RTs for the vertical LF condition, as compared with the NF condition. According to Treisman's (1985) account, search efficiency should be unchanged, or at least not slower, with the vertical frame because the orientation standard would remain vertical.

Another possibility is orientation contrast effects between the frame contour and the inside line, similar to those suggested for RF effects. Research has shown that $\mathrm{RF}$ effects increase inversely with increases in the distance between a rod and the nearest frame contour (Coren \& Hoy, 1986; Zoccolotti et al., 1993), which is taken as evidence for orientation contrast effects between the rod and the frame (Wenderoth \& Johnstone, 1987). The contrast effects are most apparent when the frame and the rod are within $1^{\circ}$ of visual angle (Zoccolotti et al., 1993). In the LF condition, the distance between the inside line and the nearest frame contour (i.e., the top and bottom component lines) was about $0.3^{\circ}$. It is thus likely that the target and distractor lines were subject to orientation contrast effects, which would have changed as a function of frame orientation (Wenderoth \& Johnstone, 1987; Zoccolotti et al., 1993). Such distortions to target and distractor orientations would lead to significant changes in search efficiency (Marendaz et al., 1993). Possible effects of orientation contrast, as well as distraction by the frame contour, were also suggested by Treisman and Gormican (1988) and Marendaz (1998) for their findings regarding frame effects.

In contrast to the remarkable finding for the LF, the effect of the GF was unimpressive. Search asymmetry was reduced by the tilted GF, but there was little change in search rate, no more than $5 \mathrm{msec} / \mathrm{item}$, from the vertical GF (Figure 2). This was much smaller than the changes of 20-30 msec/item shown in Treisman's (1985) and Marendaz's (1998) studies. Other studies, although unpublished, have also shown only moderate, if any, effects of tilted GF (C. Ballaz, personal communication, July 31, 2000; work cited by Wolfe, Friedman-Hill, Stewart, \& O'Connell, 1992). We have investigated and reported elsewhere (Mori, Kusano, \& Kataoka, 2000) reasons for the discrepant findings of GF effects in a series of experiments.

\section{EXPERIMENT 2}

In Experiment 2, we attempted to replicate the effects of LF with $18^{\circ}$-tilted lines and frames. The motive for this experiment was to answer one of the reviewers' questions concerning the generality of the LF effects observed with $30^{\circ}$ tilt angles. As has been noted, Treisman (1985) and Marendaz (1998) used an $18^{\circ}$ orientation (paired with a vertical orientation), which was found to give clear search asymmetries (Foster \& Ward, 1991a) and relatively large RF effects (Spinelli et al., 1991). We also modified other methodologiesused in Experiment 1, to eliminate factors that possibly modulated search performance and LF effects (i.e., the visible rectangular frame around the display and the jaggedness of the tilted lines). ${ }^{3}$

\section{Method}

All the stimuli were presented on a 19-in. high-resolution color monitor (Sony CPD-G420) controlled by a personal computer (Dell 
OptiPlex GX150) with a color graphic system (Cambridge Research Systems VSG2/5), which also controlled the experimental timing, RT measurement, and recording from a custom-made response box. The monitor was covered by a black board with a circular window $24.5 \mathrm{~cm}$ in diameter (visual angle of $20^{\circ}$ ), which defined the screen area. Another black board was placed between the monitor and the observer, who viewed the screen binocularly through a hole in the board that restricted the observer's view to the screen area. A chinrest was used to support the observer's head during the experiment. The experiment was conducted in a dark booth, and only the stimuli in the screen area were visible. The luminance of the stimuli was $30 \mathrm{~cd} / \mathrm{m}^{2}$. The orientation of the line items and the frames was either vertical or tilted $18^{\circ}$ left from the vertical, and anti-aliasing techniques were used to eliminate the jaggedness of the oblique lines. Other aspects of the stimuli were identical to those in the LF conditions in Experiment 1.

There were two search tasks, the target being either a tilted line or a vertical line, and three frame conditions: NF, LF vertical, and LF tilted. Unlike in Experiment 1, each subject performed both search tasks under the three conditions. Six graduate students at Tokyo Metropolitan University (3 females and 3 males, 23-27 years of age) volunteered for participation. Half of them performed the search for a tilted line first, and the other half for a vertical line first. The order of the three conditions was randomized across subjects for each task. The other procedures were identical to those in Experiment 1 .

\section{Results and Discussion}

Figure 4 shows the mean correct RTs for the present and absent trials. The results replicated the major findings of Experiment 1. In the NF condition, an asymmetry between the two search tasks was observed more clearly in the present trials than in the absent trials. ANOVAs confirmed this observation: In the present trials, there were significant main effects of target type $[F(1,5)=$ $12.13, p<.05]$ and set size $[F(3,15)=16.60, p<.01]$ and a target type $\times$ set size interaction $[F(3,15)=11.29$, $p<.01]$, whereas in the absent trial there was a significant effect of set size $[F(3,15)=12.09, p<.01]$ but no effect of target type $[F(1,5)=1.59]$ and no target type $\times$ set size interaction $[F(3,15)=1.99]$.

In the LF conditions, asymmetry between the two tasks was more marked with the vertical LF (squares), and the asymmetry was reversed with the tilted LF (diamonds). An ANOVA on the RTs for the vertical LF revealed that, among other effects, RTs were significantly longer for the vertical target than for the tilted target $[F(1,5)=60.83, p<.01]$ and showed a significantly steeper increase with increases in set size for the vertical target $[F(3,15)=57.13, p<.01]$. For the tilted LF, the results were just the opposite. RTs were significantly longer for the tilted target $[F(1,5)=276.13, p<.01]$, with a significantly steeper increase with increases in set size $[F(3,15)=78.35, p<.01]$. In comparison with the $\mathrm{NF}$ condition, searches were slower for both frame orientations. ANOVAs revealed that RTs were significantly longer for the vertical $\operatorname{LF}[F(1,5)=15.23, p<.05]$ and for the tilted $\operatorname{LF}[F(1,5)=22.53, p<.01]$, with significantly steeper increases with increases in set size [for the vertical $\operatorname{LF}, F(3,15)=12.38$; for the tilted $\operatorname{LF} F(3,15)=$ 24.48 ; both $p$ s $<.01$ ], than for the NF condition.
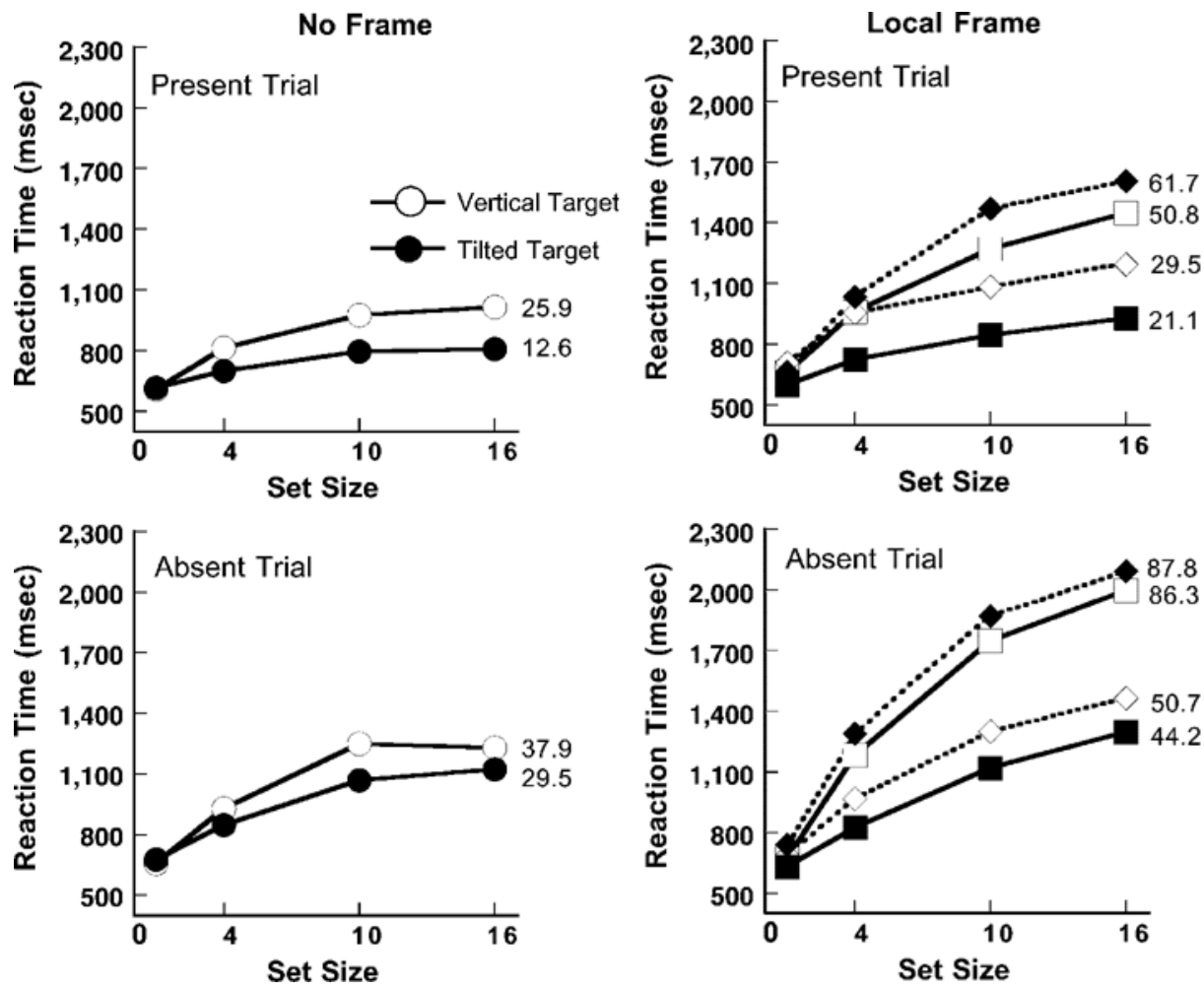

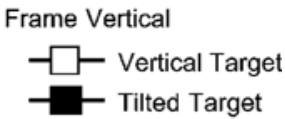

Frame Tilted --- Vertical Target Tilted Target

Figure 4. Mean correct reaction times (RTs) in Experiment 2, together with the search rates (msec/item) for the RT functions. 
Table 2

Percentages of Errors in Experiment 2

\begin{tabular}{|c|c|c|c|c|c|c|c|c|c|}
\hline \multirow[b]{2}{*}{ Frame } & \multirow[b]{2}{*}{ Target } & \multicolumn{4}{|c|}{ Present Trial } & \multicolumn{4}{|c|}{ Absent Trial } \\
\hline & & 1 & 4 & 10 & 16 & 1 & 4 & 10 & 16 \\
\hline \multirow[t]{2}{*}{ NF } & Vertical & 0.0 & 3.5 & 10.4 & 9.7 & 2.1 & 0.7 & 4.9 & 0.7 \\
\hline & Tilted & 2.1 & 1.4 & 2.1 & 5.6 & 0.7 & 2.1 & 1.4 & 0.7 \\
\hline \multirow[t]{2}{*}{ LF vertical } & Vertical & 1.4 & 1.4 & 7.6 & 32.6 & 0.7 & 0.7 & 0.7 & 5.6 \\
\hline & Tilted & 0.7 & 0.7 & 0.7 & 4.2 & 0.7 & 0.7 & 0.7 & 0.7 \\
\hline \multirow[t]{2}{*}{ LF tilted } & Vertical & 2.8 & 3.5 & 7.6 & 10.4 & 1.4 & 1.4 & 2.8 & 0.7 \\
\hline & Tilted & 0.0 & 1.4 & 19.4 & 26.4 & 2.1 & 1.4 & 2.8 & 1.4 \\
\hline
\end{tabular}

Note-NF, no frame; LF, local frame.

Table 2 presents the percentages of errors in Experiment 2. They showed a pattern similar to that observed in Experiment 1. The errors were relatively high in the present trials and increased with increases in set size for the conditions that produced slow search rates (e.g., a vertical target with the LF vertical and a tilted target with the LF tilted). The correlations between the mean RTs and the error rates were $R(22)=.864, p<.01$, in the present trials, and $R(22)=.372$, n.s., in the absent trials. For the vertical target alone, $R(10)=.799, p<.01$, in the present trials, and $R(10)=.425$, n.s., in the absent trials. For the tilted target alone, $R(10)=.927, p<.01$, in the present trials, and $R(10)=.370$, n.s., in the absent trials. These positive correlations indicate that the differences in RTs among the different frame conditions may not have been contaminated by a speed-accuracy tradeoff.

The close similarity of the results in Experiments 1 and 2 indicates that the effects of the tilted LF were not specific to the $30^{\circ}$ tilt orientation, nor were they artifacts of the methodologies used in Experiment 1, such as the jaggedness of the tilted lines and a visible rectangular board around the stimulus display.

\section{EXPERIMENT 3}

In Experiment 3, we returned to the $30^{\circ}$ tilt orientation and examined the degree to which the component lines of LF contributed to the change in search efficiency observed in Experiment 1. For this purpose, we used two variations of the LF, one made of only the left and right component lines of the LF and the other made of only the top and bottom component lines. Examples are shown in Figure 5. We refer to them as the left-and-right component (LRC) and the top-and-bottom component (TBC), respectively. The LRC was designed to test distraction effects of the left and right component lines. If these component lines served as additional distractors in Experiment 1 when their orientation matched the target orientation, the tilted LRC would produce similar effects on search efficiency. The TBC was used to examine orientation contrast effects of the top and bottom lines. If the contrast effects induced distortion of the perceived orientation of the inside line in the search task, the TBC should change the search performance from that obtained in the NF condition. In the literature on RF ef- fects, frames consisting of only a pair of parallel lines were found to induce a distortion of the apparent vertical of the rod (Wenderoth \& Beh, 1977).

\section{Method}

The LRC and the TBC consisted of a pair of parallel lines identical to the component lines of the LF in Experiment 1. The line length, as well as the separation between the two lines, was $1.3^{\circ}$ in visual angle. For the TBC, the distance between the stimulus line and either component line was approximately $0.3^{\circ}$. The NF condition, in which the stimulus lines were presented alone, was also employed. Twelve new subjects from Toyama Prefectural University (all males, 19-21 years of age) participated in this experiment for course credit. A group of 6 subjects performed the search task for the vertical line, and the other group performed the search for the tilted line. For both groups, there were five conditions involving frame type and orientation (one session for each condition): LRC vertical, LRC tilted, TBC vertical, TBC tilted, and NF. In all other respects, the method was identical to that in Experiment 1.

\section{Results}

One subject in the group for the tilted target was removed from subsequent analysis because of considerably higher error rates (more than $40 \%$ ), as compared with the other subjects. Figures 6 and 7 present the mean correct RTs for the remaining 11 subjects, separately for the target-present and target-absent trials. In the NF condition, there was an asymmetry between the search for the vertical target and the search for the tilted target, similar to the pattern in Experiment 1. An ANOVA on the mean RTs showed, among other effects, significant main effects of target type $[F(1,9)=5.75, p<.05]$ and set size $[F(3,27)=13.33, p<.01]$ and a significant interaction of target type and set size $[F(3,27)=9.08, p<$ $.01]$.

In the LRC condition, the asymmetry between the two search tasks for the tilted LRC (open and filled diamonds) was the exact inverse of that for the vertical LRC (open and filled squares). A four-factor (target type, LRC orientation, trial type, and set size) ANOVA showed no effect of target type $[F(1,9)=1.35]$ or LRC orientation $[F(1,9)=4.04]$ but a significant interaction between them $[F(1,9)=65.63, p<.01]$. For the vertical LRC, an ANOVA showed significant main effects of target type $[F(1,9)=127.08]$, trial type $[F(1,9)=76.19]$, and set size $[F(3,27)=89.07$; all $p$ s $<.01]$, and their interactions were all significant, with $p$ s $<.01$. For the tilted LRC, there were significant main effects of target 
(A)

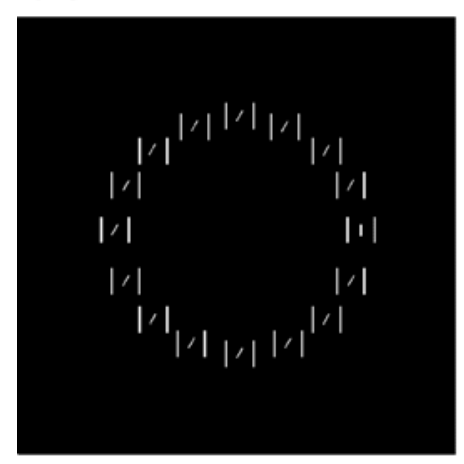

(C)

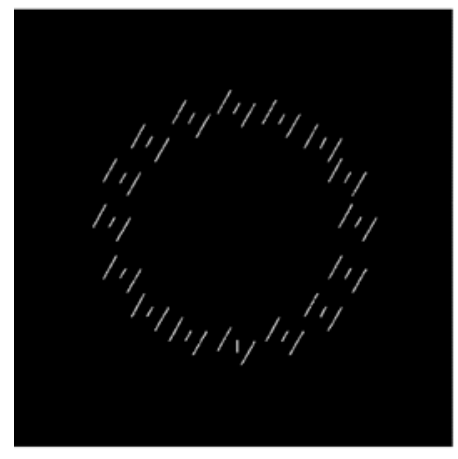

(E)

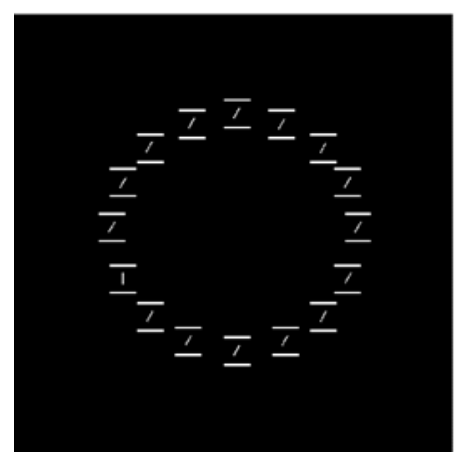

(B)

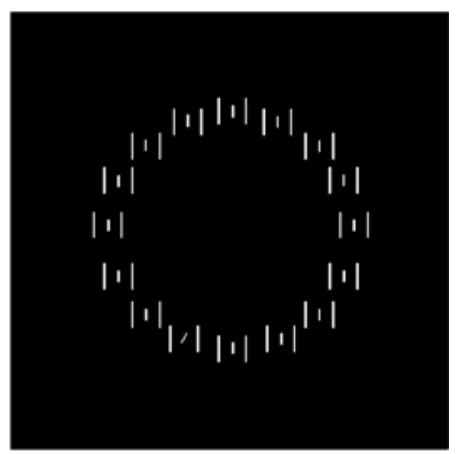

(D)

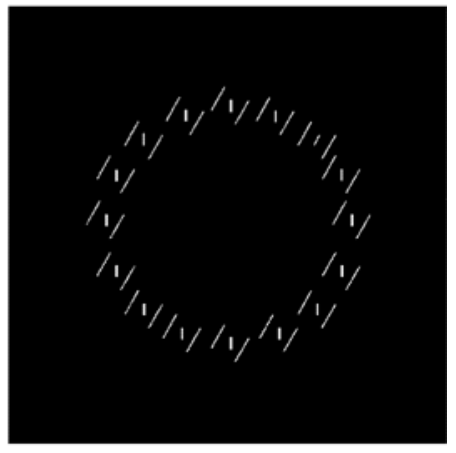

(F)

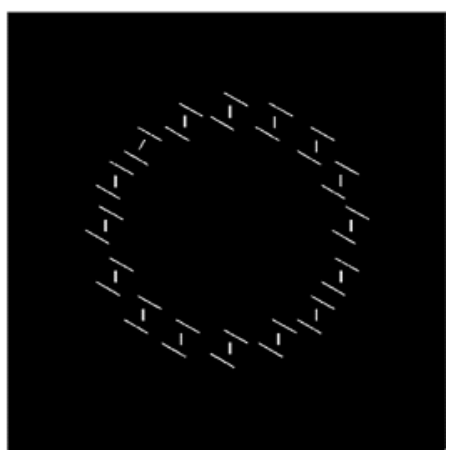

Figure 5. Examples of search displays in Experiment 3. All the examples represent 16-item target-present trials, the left ones showing the search for a vertical target and the right ones for a tilted target. Panels $A$ and $B$ are vertical leftand-right-component (LRC) local frames (LFs), panels $C$ and $D$ are tilted LRCs, and panels $E$ and $F$ are vertical and tilted top-and-bottom component (TBC) LFs, respectively.

type $[F(1,9)=6.19, p<.05]$, trial type $[F(1,9)=40.31$, $p<.01]$, and set size $[F(3,27)=24.90, p<.01]$ and a significant interaction of trial type and set size $[F(3,27)=15.47, p<.01]$. The lack of a main effect of target type in the overall ANOVA seems to have been due to the opposing patterns of asymmetry produced by the two component orientations, which canceled out each other's effects. In comparison with the NF condition, the vertical LRC yielded a larger asymmetry between the two tasks, with a slower search for the vertical target. ANOVAs showed that search for the vertical target was significantly slower for the vertical LRC than for the NF condition $[F(1,5)=27.35, p<.01]$, whereas the search for the tilted target did not differ between the two conditions $[F(1,5)=0.06]$.

In the TBC condition, there was a clear asymmetry between the two search tasks with the vertical TBC, but the asymmetry was much reduced with the tilted TBC, par- 

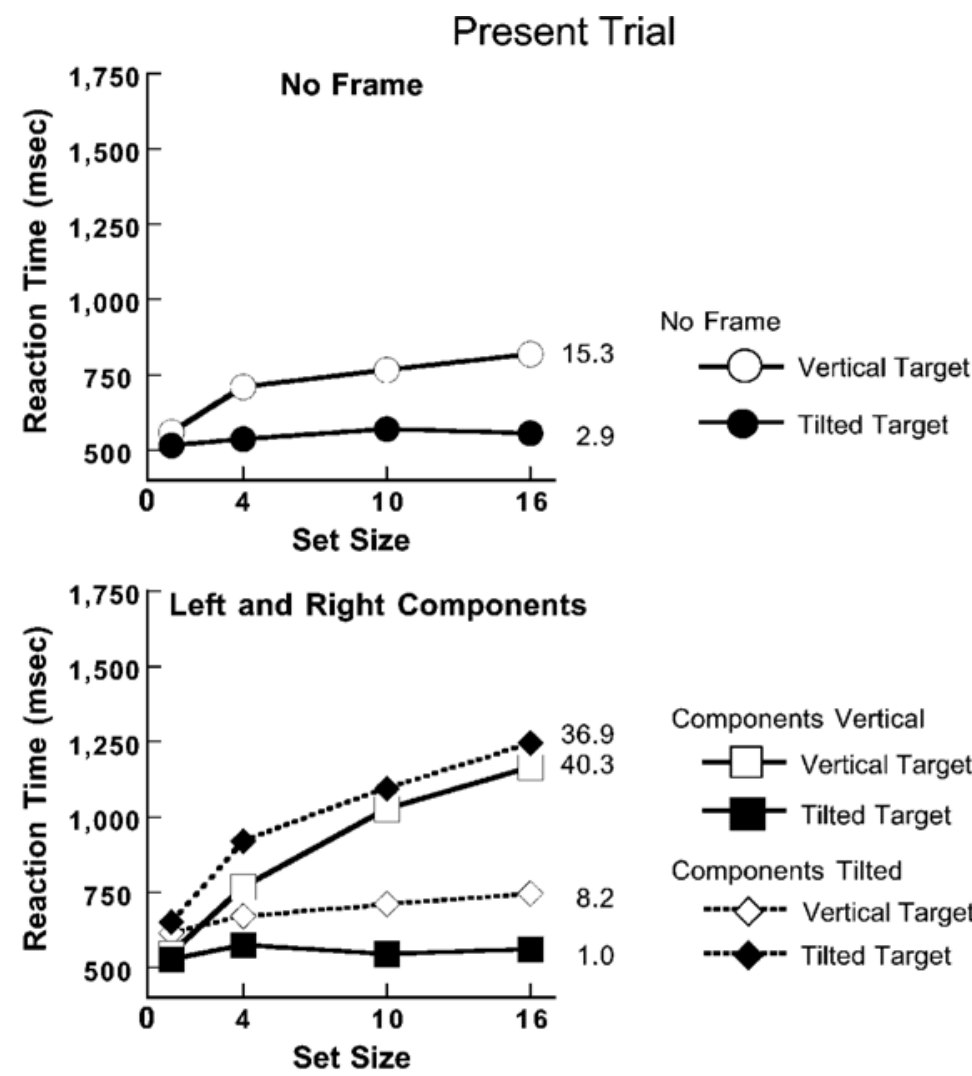

Components Vertical
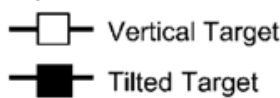

Components Tilted

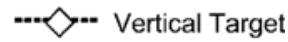

Tilted Target

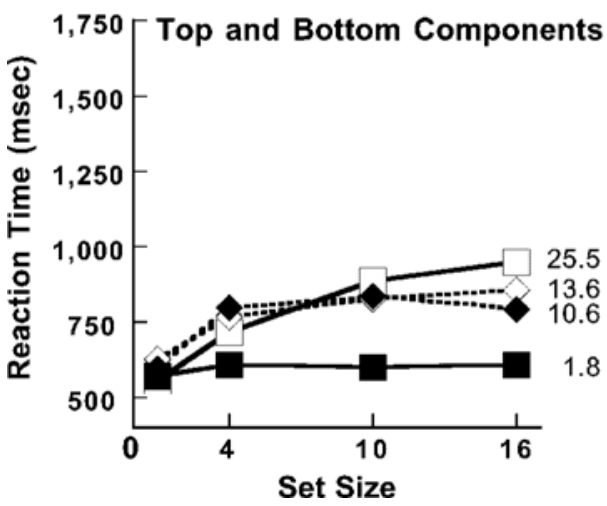

Figure 6. Mean correct reaction times (RTs) in the present trials in Experiment 3 , together with the search rates $(\mathrm{msec} / \mathrm{item})$ for the RT functions.

ticularly in the present trials. A four-factor ANOVA showed, among other effects, significant main effects of target type $[F(1,9)=5.54, p<.05]$ and TBC orientation $[F(1,9)=6.21, p<.05]$ and a significant interaction between them $[F(1,9)=10.69, p<.01]$. For the vertical TBC, there were significant main effects of target type $[F(1,9)=13.15, p<.01]$, trial type $[F(1,9)=28.08$, $p<.01]$, and set size $[F(3,27)=68.87, p<.01]$, and their interactions were all significant, with $p<.05$ or less. For the tilted TBC, there were significant main effects of trial type $[F(1,9)=74.49, p<.01]$ and set size $[F(3,27)=30.47, p<.01]$, but neither the effect of tar- get type $[F(1,9)=0.63]$ nor the interaction of target type and set size $[F(3,27)=1.39]$ was significant. The lack of these effects indicates that search asymmetry was reduced by the tilted TBC. In comparison with the NF condition, the vertical TBC yielded a slower search for the vertical target. Although there was no significant difference in RTs between the two conditions $[F(1,5)=5.19]$, the increase in RT with increases in set size was significantly steeper for the vertical TBC $[F(3,15)=63.91$, $p<.01]$. RTs for the tilted target did not show a significant difference between the two conditions $[F(1,4)=$ $2.80]$ or an interaction with set size $[F(3,12)=3.22]$. 

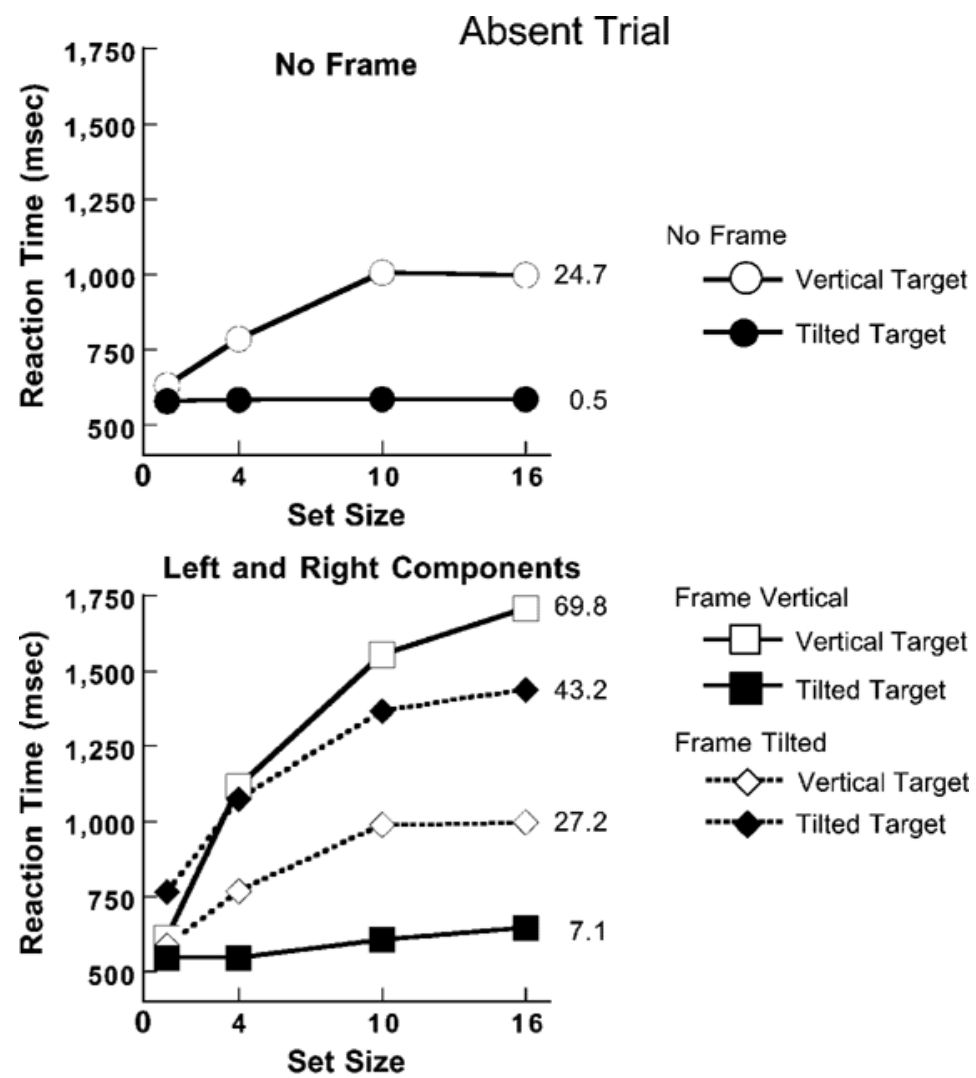

Frame Tilted
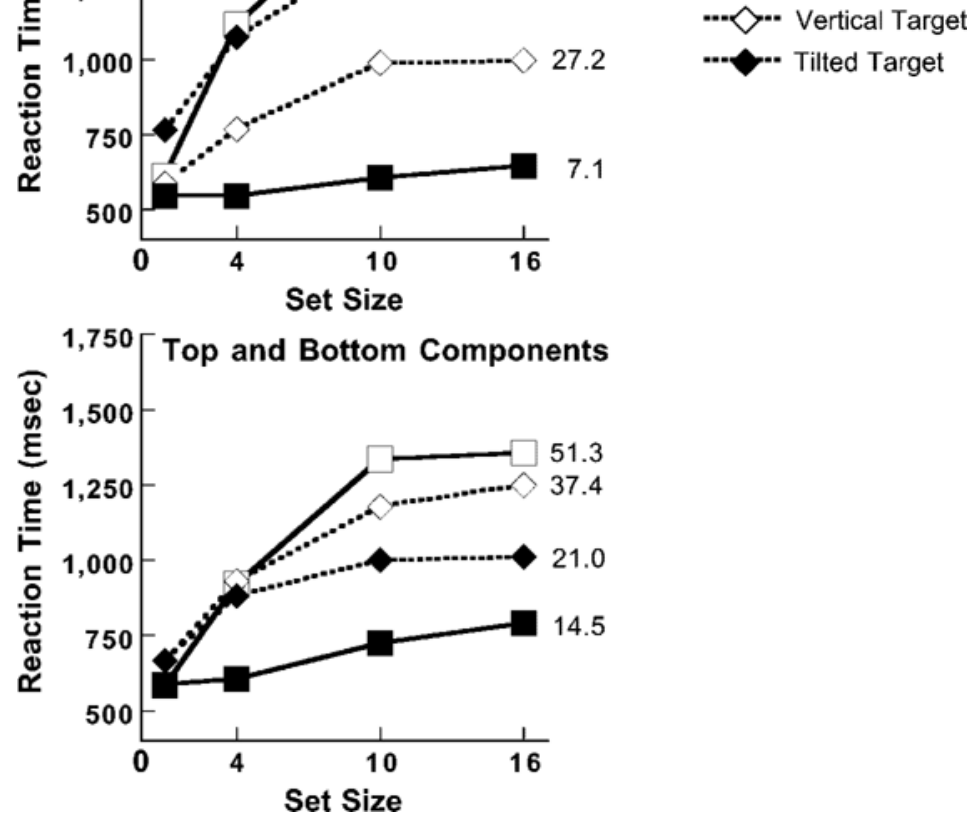

Figure 7. Mean correct reaction times (RTs) in the absent trials in Experiment 3, together with the search rates (msec/item) for the RT functions.

Table 3 presents the percentages of errors in Experiment 3. Two points are noteworthy. First, the error rates in the NF condition were higher for the tilted target than for the vertical target. Since RTs were shorter for the tilted target, the difference in RTs may reflect, at least partly, a speed-accuracy tradeoff. Second, the error rates for 10 and 16 items were considerably higher in the LRC condition when the frame orientation matched the target orientation (e.g., a vertical LRC and a vertical target or a tilted LRC and a tilted target). In other respects, the pattern of error rates was generally consistent with that in Experiment 1. The correlations between the mean RTs and the error rates were $R(38)=.700, p<.01$, in the pres- ent trials, and $R(38)=.229$, n.s., in the absent trials. For the vertical target alone, $R(18)=.762, p<.01$, in the present trials, and $R(18)=.552, p<.05$, in the absent trials. For the tilted target alone, $R(18)=.869, p<.01$, in the present trials, and $R(18)=.525, p<.05$, in the absent trials. These positive correlations suggest that the differences in RTs among the different frame conditions were not contaminated by a speed-accuracy tradeoff.

\section{Discussion}

The reversal of search asymmetry by the tilted LRC clearly supports the possibility that the left and right component lines serve as additional distractors in target 
Table 3

Percentages of Errors in Experiment 3

\begin{tabular}{|c|c|c|c|c|c|c|c|c|c|}
\hline \multirow[b]{2}{*}{ Frame } & \multirow[b]{2}{*}{ Target } & \multicolumn{4}{|c|}{ Present Trial } & \multicolumn{4}{|c|}{ Absent Trial } \\
\hline & & 1 & 4 & 10 & 16 & 1 & 4 & 10 & 16 \\
\hline \multirow[t]{2}{*}{$\mathrm{NF}$} & Vertical & 1.4 & 0.7 & 3.5 & 7.6 & 2.8 & 4.2 & 5.6 & 2.8 \\
\hline & Tilted & 12.5 & 9.2 & 7.5 & 15.8 & 10.8 & 9.2 & 10.0 & 15.8 \\
\hline \multirow[t]{2}{*}{ LRC vertical } & Vertical & 0.0 & 1.4 & 12.5 & 34.0 & 1.4 & 9.7 & 6.9 & 9.7 \\
\hline & Tilted & 1.7 & 5.0 & 1.7 & 2.5 & 5.8 & 1.7 & 5.8 & 4.2 \\
\hline \multirow[t]{2}{*}{ LRC tilted } & Vertical & 6.3 & 1.4 & 1.4 & 4.2 & 0.0 & 0.7 & 1.4 & 2.8 \\
\hline & Tilted & 5.0 & 11.7 & 29.2 & 46.7 & 9.2 & 8.3 & 19.2 & 17.5 \\
\hline \multirow[t]{2}{*}{ TB vertical } & Vertical & 0.7 & 2.1 & 4.2 & 6.3 & 3.5 & 2.1 & 2.8 & 0.7 \\
\hline & Tilted & 3.3 & 5.0 & 5.0 & 5.8 & 5.0 & 2.5 & 5.8 & 6.7 \\
\hline \multirow[t]{2}{*}{ TB tilted } & Vertical & 1.4 & 0.0 & 2.1 & 3.5 & 2.1 & 1.4 & 4.2 & 2.1 \\
\hline & Tilted & 5.8 & 15.8 & 11.7 & 14.2 & 4.2 & 6.7 & 4.2 & 3.3 \\
\hline
\end{tabular}

Note-NF, no frame; LRC, left-to-right components; TB, top-to-bottom components.

detection. Given the similar patterns of results for the LRC and the LF, one might argue that the effects of the tilted LF resulted solely from distraction by the left and right component lines. Although we believe that those component lines made a significant contribution to the effects of the LF, we are also concerned that the effects of the LRC obtained here might partially have been artifacts from the use of two line segments as a frame. Our concern is based on the error rates for the target-present trials (Table 3): The error rates were very high for 10 and 16 items when the target and the LRC were of the same orientation. In contrast, the error rates were low when the target and the LRC were different in orientation. A similar pattern was observed in the LF condition in Experiment 1 (Table 1), but the differences across target and frame orientations were more marked in the LRC condition. We suspect that target detection was more difficult in the LRC condition than in the LF condition when the target and the LRC orientations were the same. Since the LRC was made of line segments that were just like the target line, the LRC would have been more difficult to discriminate from the target line than was the LF. So the LRC may have generated much larger distraction than that caused by the full-contour LF.

The results for the TBC suggest that the orientation contrast induced distortion to orientation perception of the inside lines and, as a result, to search performance, in the following way. Orientation contrast is a function of angular difference between lines (Carpenter \& Blakemore, 1973; Wenderoth \& Johnstone, 1987). In this experiment (and Experiment 1), the angular difference between the $\mathrm{TBC}$ and the inside line was either $60^{\circ}$ (when the TBC and the line differed in orientation) or $90^{\circ}$ (when the TBC and the line matched in orientation). Previous studies have shown that there is little contrast effect between lines differing by $90^{\circ}$ (Over, Broerse, \& Crassini, 1972; Wenderoth \& Beh, 1977). So we assume that the contrast effect occurred only for the $60^{\circ}$ difference between the inside line and the TBC: The line was perceived as less slanted with respect to the TBC. ${ }^{4}$ In the presence of the vertical TBC, the tilted lines were subject to the contrast effect. In the search for the vertical target, the distractor (tilted) lines were perceived as less tilted than they actually were, making the discrimination between the target and the distractor lines more difficult than when the lines were presented alone. As a result, search was slower ( $25.5 \mathrm{msec} / \mathrm{item})$, as compared with the NF condition ( $15.3 \mathrm{msec} / \mathrm{item})$. In the search for the tilted target, the target was perceived as less tilted. However, search for the tilted target was less susceptible to the orientation of the target as long as the target was categorized as tilted among vertical distractors (Wolfe, 1999; Wolfe et al., 1992). So search was still efficient (1.8 msec/item), as in the NF condition ( $2.9 \mathrm{msec} / \mathrm{item})$. With the tilted TBC, the vertical lines were perceived as tilted, due to the contrast effect. In search for the vertical target, the target were perceived as tilted. In search for the tilted target, the distractors were perceived as tilted. Both search tasks became searches for a tilted target among more or less tilted distractors, and search was equally efficient in both tasks (13.6 and $10.6 \mathrm{msec} / \mathrm{item}$ ).

Therefore, this experiment provided clear evidence that distraction and orientation contrast by frame components significantly contribute to the effects of the LF. The next question was whether a shift in the reference standard, as was suggested in the discussion of Experiment 1 , would also be implicated in the effects of the LF or whether distraction and an orientation contrast would suffice to explain such effects. In Experiment 4, this question was addressed.

\section{EXPERIMENT 4}

In Experiment 4, we used two frame orientations, $15^{\circ}$ and $45^{\circ}$ right from the vertical, in separate sessions. For both frame orientations, the target and the distractor lines were either vertical or tilted $30^{\circ}$, identical to those in Experiments 1 and 3 . According to the reference standard account (Treisman, 1985), both vertical and $30^{\circ}$-tilted lines would be positively coded when the LF was tilted either $15^{\circ}$ or $45^{\circ}$, because the two lines would deviate from the standard, which would be aligned by the LF orientation. This means that whichever of the lines was used as a target, the target (and the distractors) would signal the positive value in the display, and the target detection should be fast (Treisman, 1985; Treisman \& 
Gormican, 1988). In line with this logic, it was predicted that searches for a vertical target and for a tilted target would become equally fast in the $15^{\circ}$-tilted and $45^{\circ}$-tilted LF conditions, possibly at a level comparable to that for a search for a tilted target in the NF condition.

For the $15^{\circ}$-tilted and $45^{\circ}$-tilted LFs, neither distraction nor orientation contrast from the frame side would be involved in search performance. The orientation of the left and right component lines would be different from the orientations of the inside lines (vertical and $30^{\circ}$-tilted) by more than $15^{\circ}$, so these component lines should not act as additional distractors in the search for either line. It is also unlikely that the top and bottom component lines of either frame orientation would induce strong contrast effects. Figure 8 illustrates the angular relations of the LFs and the inside lines. For the $15^{\circ}$-tilted LF, the top and bottom component lines were different by $75^{\circ}$ from either the vertical or the tilted lines (see Figures $8 \mathrm{~A}$ and $8 \mathrm{~B}$ ), and the $75^{\circ}$ separation was too large to induce orientation contrast (Over et al., 1972; Wenderoth \& Beh, 1977). For the $45^{\circ}$-tilted frame, the angular difference from the component lines was $45^{\circ}$ for the vertical line and $75^{\circ}$ for the tilted line (Figures 8C and 8D). Note that the vertical line formed a $45^{\circ}$ angle with the two neighboring component lines, so that the contrast effects of these component lines would cancel each other out.

\section{Method}

The frames were identical to the LF in Experiment 1, except for the orientation, which was $15^{\circ}$ or $45^{\circ}$ right from the vertical. The two frame orientations were used in separate sessions. There were
(A) $15^{\circ}$-tilted LF

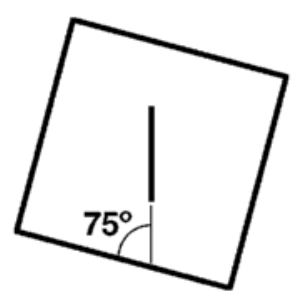

(C) $45^{\circ}$-tilted LF Vertical Line

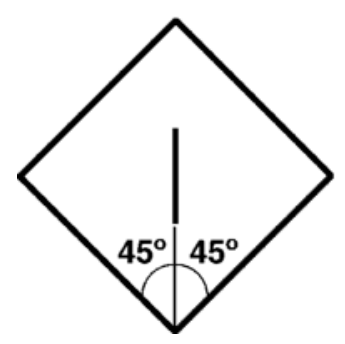

(B) $15^{\circ}$-tilted LF Tilted Line

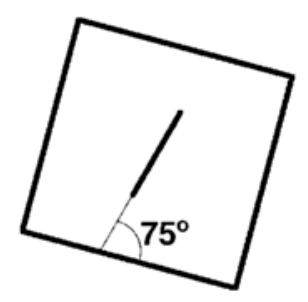

(D) $45^{\circ}$-tilted LF Tilted Line

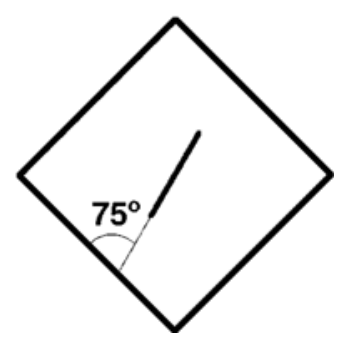

Figure 8. Illustrations of $15^{\circ}$-tilted and $45^{\circ}$-tilted local frames (LFs) used in Experiment 4. In each figure, the angle between the inside line and the bottom component line of the frame is shown.
12 new subjects in this experiment (all males, 19-21 years of age), 6 of them performing the task for the vertical target and the other 6 performing the task for the tilted target. For both groups, the experiment consisted of three conditions (one session for each condition): $15^{\circ}$-tilted LF, $45^{\circ}$-tilted LF, and NF. In all other respects, the method was identical to that in Experiment 3.

\section{Results}

Figure 9 shows the mean correct RTs in this experiment. Unlike the previous experiments, there was no asymmetry between the two search tasks in the NF condition. An ANOVA showed no effect of target type $[F(1,10)=0.0]$, and only the main effects of trial type $[F(1,10)=14.42]$ and set size $[F(3,30)=14.65]$ were significant (both $p \mathrm{~s}<.01$ ).

In the LF condition, the searches for the two types of targets became equally slower for the $15^{\circ}$ and the $45^{\circ}$ frame orientations, in direct contrast to the prediction of the reference standard hypothesis. An ANOVA showed significant effects of frame orientation $\left[15^{\circ}\right.$ or $45^{\circ}$, $F(1,10)=5.72, p<.05]$, trial type $[F(1,10)=48.35$, $p<.01]$, and set size $[F(3,30)=111.49, p<.01]$ and a significant interaction of trial type and set size $[F(3,30)=$ $24.32, p<.01]$, but there was no significant effect of target type $[F(1,10)=1.94]$ and no other interactions. Subsequent analyses showed that the significant effect of frame orientation reflected the difference in the absent trials, in which RTs were significantly longer for the $15^{\circ}$-tilted LF than for the $45^{\circ}$-tilted $\operatorname{LF}[F(1,10)=6.06$, $p<.05]$. There was no significant difference in the present trials $[F(1,10)=2.92]$. In comparison with the NF condition, both frame orientations yielded significantly longer RTs $\left[F(1,10)=204.00\right.$ for the $15^{\circ}$-tilted LF and $F(1,10)=202.36$ for the $45^{\circ}$-tilted LF; both $p$ s $\left.<.01\right]$ and significantly steeper increases in set size $[F(3,30)=$ 72.17 for the $15^{\circ}$-tilted LF and $F(3,30)=103.89$ for the $45^{\circ}$-tilted LF; both $p$ s $\left.<.01\right]$.

Table 4 presents the percentages of errors in this experiment. The correlations between the mean RTs and the error rates were $R(22)=.722$ in the present trials, and $R(22)=.779$ in the absent trials (both $p$ s $<.01$ ). For the vertical target, $R(10)=.737$ in the present trials, and $R(10)=.739$ in the absent trials (both $p \mathrm{~s}<.01$ ). For the tilted target, $R(10)=.748$ in the present trials, and $R(10)=.825$ in the absent trials (both $p \mathrm{~s}<.01$ ). As in the previous experiments, the differences in RTs among the frame conditions may not have been contaminated by a speed-accuracy tradeoff.

\section{Discussion}

The results in this experiment do not support the reference standard hypothesis, which predicts fast searches in the $15^{\circ}$ - and $45^{\circ}$-tilted LF conditions. Rather, the searches became much slower in those two conditions than in the NF condition. The results also differ from those in Marendaz's (1998) study, which showed that a $36^{\circ}$-tilted frame that surrounded an entire display did not affect search for either a vertical target or an $18^{\circ}$-tilted target. Remember that the reference standard account 

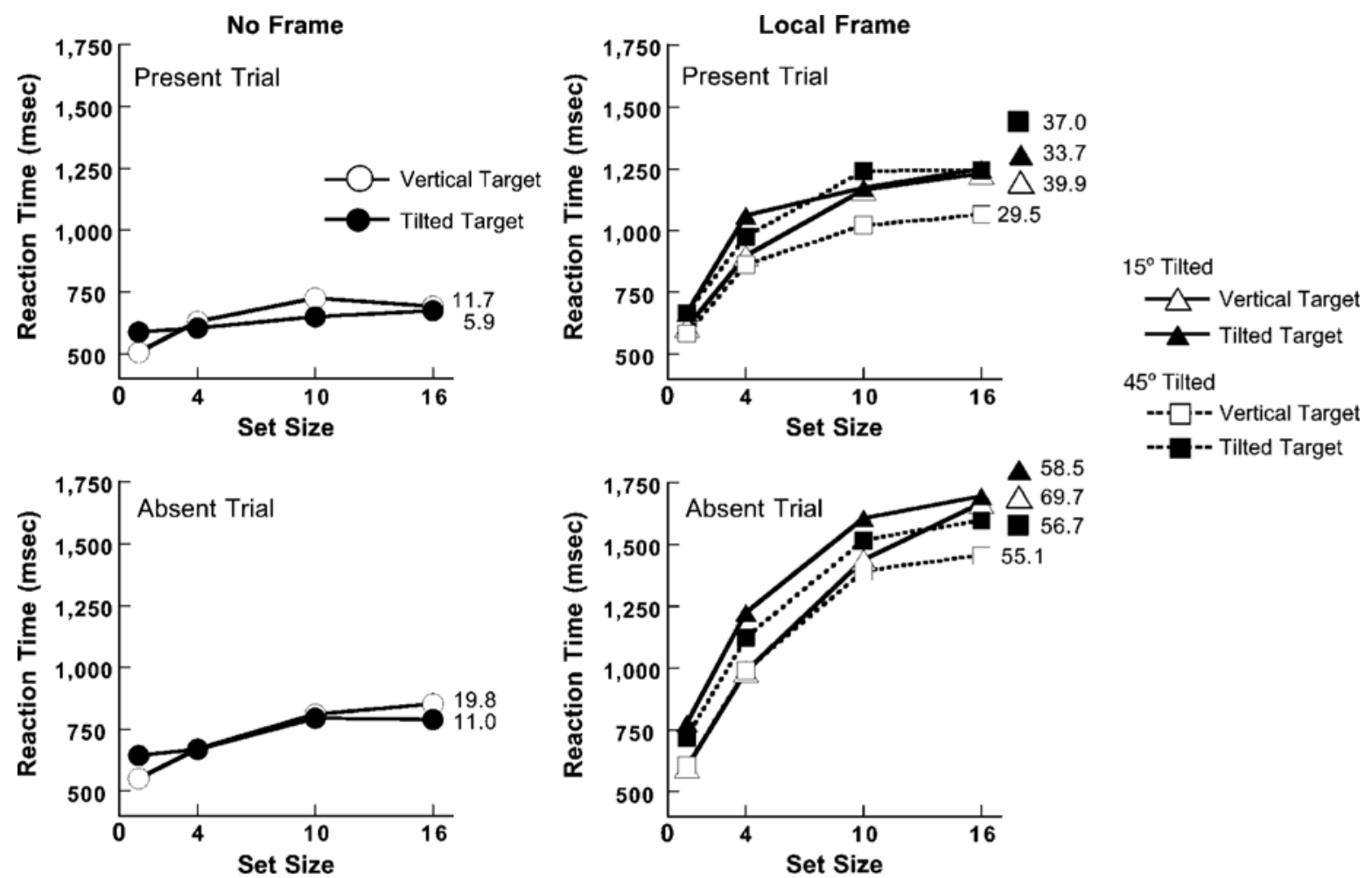

Figure 9. Mean correct reaction times (RTs) in Experiment 4, together with the search rates (msec/item) for the RT functions.

was originally proposed by Treisman (1985) to explain her results for a frame surrounding an entire display. Treisman (1985) and Marendaz both argued that the frame operates on a reference system for the coding of line orientation. The results of this experiment suggest that the LF acts differently from the operation of the frame used in their studies.

We also reasoned that there would be little effect of either distraction or orientation contrast from the $15^{\circ}$ - and $45^{\circ}$-tilted LFs. So what kind of operation do those LFs have that caused the slow searches in this experiment? One suggestion comes from a recent study by Hayward and Burke (2000). They found that search for a tilted line among vertical distractors became very slow when each line item was surrounded by a small circle, in a manner similar to that in the LF condition in the present study.
When the lines were located outside the circles, the search was as fast as when the lines were presented alone. Hayward and Burke suggested that the circles, when they surrounded the lines, interfered in the orientation processing of the neighboring lines that played an important role in searching for a target line (Nothdurft, 1993).

Following Hayward and Burke (2000), we hypothesize that LFs, like circles surrounding lines, interfere in the orientation processing of neighboring items or a group of nearby items in the display. Such orientation processing includes local orientation contrast and perceptual grouping, both of which are known to mediate visual search for line orientation (Bacon \& Egeth, 1991; Nothdurft, 1991, 1992, 1993). A series of studies by Nothdurft $(1991,1992$, 1993) demonstrated that orientation differences between

Table 4

Percentages of Errors in Experiment 4

\begin{tabular}{|c|c|c|c|c|c|c|c|c|c|}
\hline \multirow[b]{2}{*}{ Frame } & \multirow[b]{2}{*}{ Target } & \multicolumn{4}{|c|}{ Present Trial } & \multicolumn{4}{|c|}{ Absent Trial } \\
\hline & & 1 & 4 & 10 & 16 & 1 & 4 & 10 & 16 \\
\hline \multirow[t]{2}{*}{$\mathrm{NF}$} & Vertical & 2.8 & 3.5 & 6.9 & 6.3 & 4.2 & 1.4 & 1.4 & 2.8 \\
\hline & Tilted & 2.1 & 0.7 & 0.7 & 4.2 & 3.5 & 2.1 & 2.1 & 0.7 \\
\hline \multirow[t]{2}{*}{ LF $15^{\circ}$-tilted } & Vertical & 3.5 & 5.6 & 8.3 & 15.3 & 0.7 & 1.4 & 4.9 & 6.9 \\
\hline & Tilted & 4.9 & 4.9 & 6.9 & 17.4 & 5.6 & 4.9 & 11.1 & 15.3 \\
\hline \multirow[t]{2}{*}{ LF $45^{\circ}$-tilted } & Vertical & 2.1 & 11.8 & 16.7 & 26.4 & 1.4 & 3.5 & 4.2 & 11.1 \\
\hline & Tilted & 4.2 & 2.8 & 13.9 & 29.9 & 4.9 & 4.9 & 6.3 & 8.3 \\
\hline
\end{tabular}

Note-NF, no frame; LF, local frame. 
neighboring lines, or a local orientation contrast, affect search performance. Search becomes increasingly efficient as orientation contrast between a target and an adjacent distractor increases and/or contrast between neighboring distractors decreases. Perceptual grouping of distractor items also facilitates search performance, by allowing an observer to process more than one item at a single attentional glance, so that less time is required for processing all the items in the display than when search operates item by item (Humphreys, Quinlan, \& Riddoch, 1989; Pashler, 1987; Wolfe, 1999). Research has shown that grouping is based on proximity and similarity of items (Bacon \& Egeth, 1991; Duncan \& Humphreys, 1989). For line orientation, Bacon and Egeth provided evidence that grouping of adjacent distractor lines (which were of identical orientation and size) improved search performance.

In the present experiments, local orientation contrast and perceptual grouping were most likely to have been at work in the NF condition, in which all distractor lines were of identical orientation and size. The regularity of item distribution (with no jitter added to item positions) may have enhanced their operation (Humphreys et al., 1989; Meigen, Lagreze, \& Bach, 1994). This inference is supported by the present finding of a relatively fast search for a vertical line (e.g., Experiment 2), as compared with the search rates reported in the previous studies (e.g., Marendaz, 1998; Treisman, 1985). Surrounding each line in the display, LFs would interfere in the orientation processing of adjacent lines, resulting in a slow search, as compared with performance with no frame. This accounts for the results of this experiment and for some of the results in Experiments 1 and 2. In Experiment 1, for example, the search for a tilted target was slower in the vertical LF than in the NF condition. This result was not due to the effects of the frame components; in Experiment 3, the search for a tilted target was as fast in the vertical LRC and TBC conditions as in the NF condition (see Figures 6 and 7).

The interference hypothesis provides a likely explanation for the relatively slow search observed in the LF conditions, yet some verification is needed for the effect. This experiment showed that with the tilted LFs, searches for a vertical target and for a tilted target were equally slower than performance in the NF condition, which seems to imply that interference effects were not different between the two search tasks. However, it should be remembered that the performance in the NF condition was atypical, showing no asymmetry between the two search tasks (cf. Experiments 1 and 2). Therefore, possible task dependency of the interference effect needs to be checked in further experiments that show search asymmetries with no LF present. It is also necessary to see whether and to what degree search performance will be affected by interference from the vertical and $30^{\circ}$-tilted LFs, such as those used in Experiment 1 , which match the orientation of the inside line. A problem in testing those LFs is that they also cause distraction and orientation contrast, which would be confounded by interference effects. In the next two experiments, we attempted to resolve this problem by modifying the shape of the square frame (see Figures 10 and 12).

\section{EXPERIMENT 5}

In Experiment 5, we used squares with gaps as LFs. Examples are shown in Figure 10. The gapped frame had the virtual contour of a square, but its left and right component lines were clearly discernible from the inside lines, because of the gaps. So these component lines would not have acted as additional distractors, unlike the full-contour LF (Experiments 1 and 2) and the LRC (Experiment 3 ). It is also unlikely that the top and bottom component lines would have induced strong contrast effects, because the gap kept the component lines from forming a clear angle with the inside line. In a study of RF effects, Streibel et al. (1980) used a similar gapped square as a surrounding frame and found that the square induced a change in the apparent orientation of the rod, although the size of the change was smaller than that caused by the full-contour square.

\section{Method}

The frame used was identical to the LF in Experiment 1, except for a gap at each side. The gap was half the size of the frame side, approximately $0.6^{\circ}$ of visual angle. Twelve new subjects from Toyama Prefectural University (all males, 19-21 years of age) participated in this experiment, a group of 6 subjects performing the search task for the vertical target and the other group performing the search task for the tilted target. For both groups, three conditions were employed (one session for each condition): LF vertical, LF tilted, and NF. In all other respects the method was identical to that in Experiments 3 and 4.

\section{Results}

Figure 11 shows the mean correct RTs for the present and absent trials. In the NF condition, there was an asymmetry between the two search tasks, similar to the results of Experiments 1 and 3. An ANOVA showed significant main effects of target type $[F(1,10)=22.52]$, trial type $[F(1,10)=21.00]$, and set size $[F(3,30)=18.23$; all $p \mathrm{~s}<.01]$ and a significant interaction of target type and set size $[F(3,30)=9.33, p<.01]$.

In comparison with the NF condition, both vertical and tilted LFs produced significantly slower searches for the vertical target $[F(1,5)=80.57$ for the vertical $L F$ and $F(1,5)=69.10$ for the tilted LF] and for the tilted target $[F(1,5)=17.93$ for the vertical $L F$ and $F(1,5)=217.79$ for the tilted LF; all $p s<.01]$. However, the pattern of results was quite different between the two frame orientations. For the vertical LF, there was a clear asymmetry between the two tasks, more marked than the asymmetry of the NF condition. For the tilted LF, the asymmetry was reversed but the difference between the two tasks was small. A four-factor ANOVA showed, among other effects, significant main effects of target type $[F(1,10)=8.78, p<$ $.05]$, frame orientation $[F(1,10)=26.80, p<.01]$, and set size $[F(3,30)=343.82, p<.01]$ and significant in- 
(A)

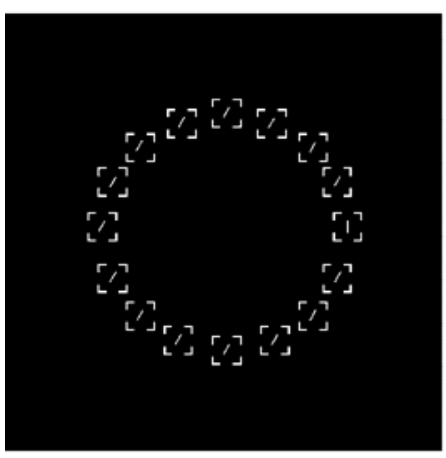

(C)

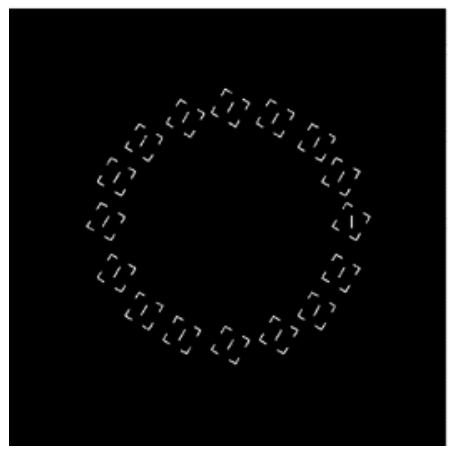

(B)

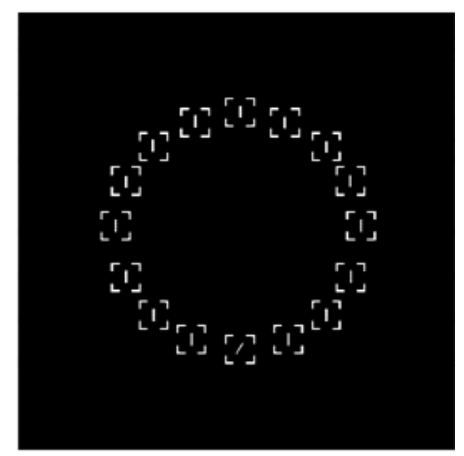

(D)

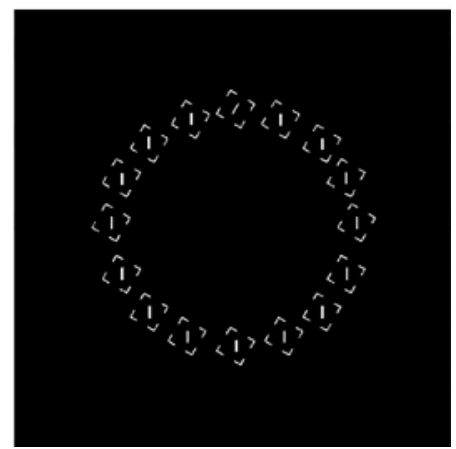

Figure 10. Examples of search displays in Experiment 5. All the examples represent 16-item target-present trials, the left ones showing the search for a vertical target and the right ones, for a tilted target. Panels $A$ and $B$ show vertical local frames (LFs), and panels C and D show tilted LFs.

teractions of target type and frame orientation $[F(1,10)=$ $37.64]$, target type and set size $[F(3,30)=7.45]$, frame orientation and set size $[F(3,30)=11.31]$, and target type, frame orientation, and set size $[F(3,30)=24.54$; all $p$ s $<.01]$. Subsequent ANOVAs showed that for the vertical LF, search for the vertical target was significantly slower $[F(1,10)=29.22, p<.01]$ and increased more rapidly with increases in set size $[F(3,30)=34.57, p<$ .01] than search for the tilted target. For the tilted LF, there was no significant effect of target type $[F(1,10)=1.41]$ and no target type $\times$ set size interaction $[F(3,30)=2.32]$.

Table 5 presents the percentages of errors in this experiment. As was observed in Experiment 3, the error rates were higher when the target and the frame were matched in orientation than when they were not. The correlations between the mean RTs and the error rates were $R(22)=.836, p<.01$, in the present trials, and $R(22)=$ .381 , n.s., in the absent trials. For the vertical target alone, $R(10)=.862$ in the present trials, and $R(10)=$ .813 in the absent trials (both $p \mathrm{~s}<.01$ ). For the tilted target, $R(10)=.858, p<.01$, in the present trials, and $R(10)=.065$, n.s., in the absent trials. Therefore, the differences in RTs among the frame conditions may not have been contaminated by a speed-accuracy tradeoff.

\section{Discussion}

The relatively slow search in the LF conditions, as compared with performance in the NF condition, is consistent with the results of the previous experiments and supports the possibility that both vertical and $30^{\circ}$-tilted LFs interfere in orientation processing of adjacent distractor lines and slow down search performance. Unlike the results of Experiment 4, the degree to which the search was affected by those LFs varied with their orientation, as well as with the orientation of the target line. For the vertical LF, the search for the vertical target was slowed to a larger degree than the search for the tilted target, showing a large asymmetry between them. For the tilted LF, the asymmetry was reversed but small and insignificant. The small asymmetry might have been due to distraction caused by the virtual contour of the gapped LF, which would have a weaker effect than the full-contour frame used in Experiment 1 (Streibel et al., 1980).

\section{EXPERIMENTS 6A AND 6B}

A final set of experiments involved frames with circular contours. Examples are shown in Figure 12. In Experiment $6 \mathrm{~A}$, we used square frames of which the inner 

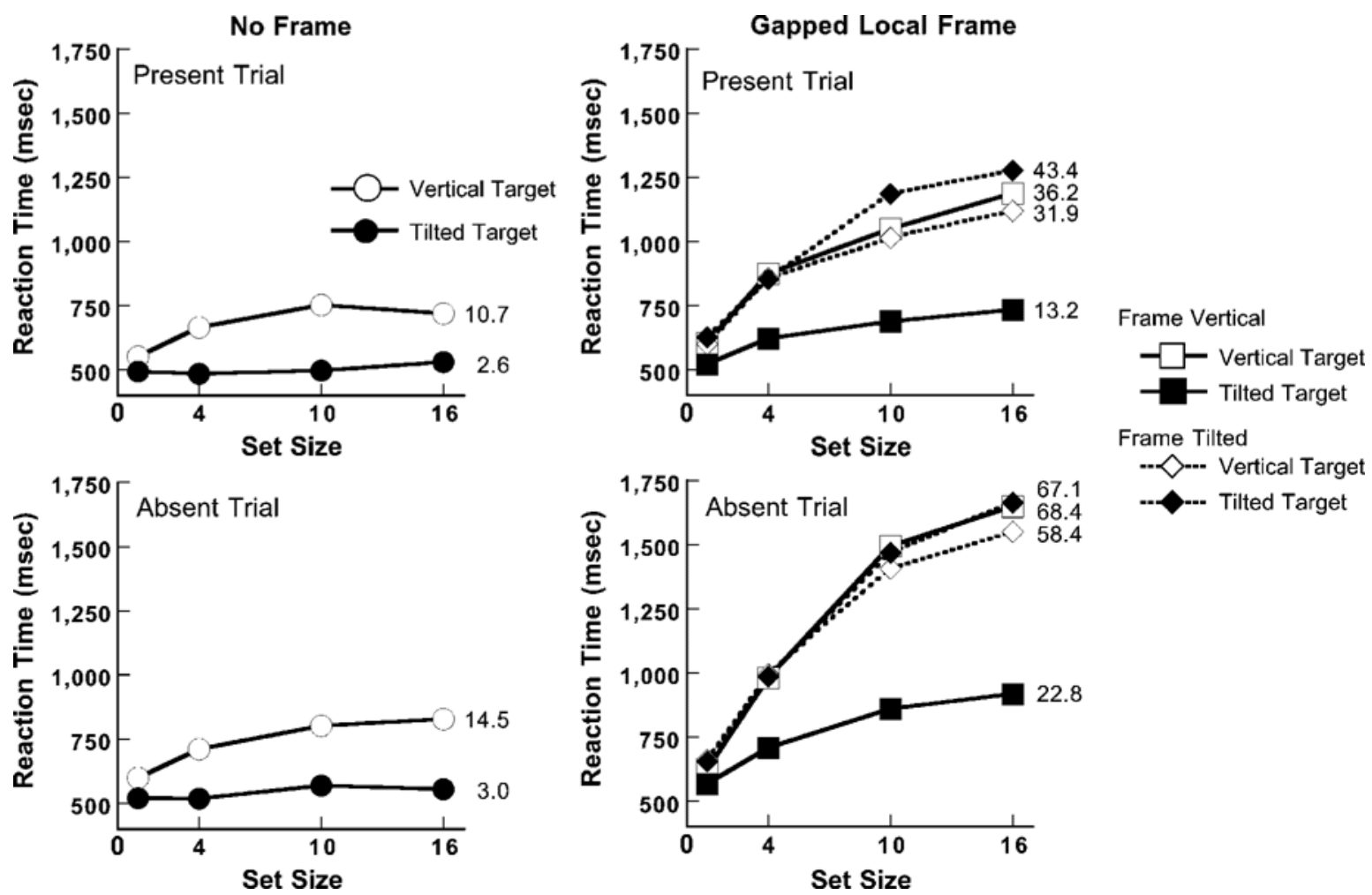

Figure 11. Mean correct reaction times (RTs) in Experiment 5, together with the search rates (msec/item) for the RT functions.

contours were circular (Figures 12A and 12B). This shape would be more effective in negating distraction and contrast effects of the frame sides than would the gapped LF used in Experiment 5. In Experiment 6B, we used circles (Figure 12C), such as those used in the study by Hayward and Burke (2000), and square frames that were identical in shape to those used in Experiment 6A, except that they were tilted $45^{\circ}$ from the vertical (Figure 12D). These $45^{\circ}$ frames were not aligned with the inside lines, nor did they give a strong sense of tiltedness or verticalness. We expected these two frames to demonstrate a genuine form of interference and to serve as control conditions for Experiment 5 and Experiment 6A, to allow us to see how the interference effect would be altered by frame orientation.

\section{Method}

The apparatus was identical to that used in Experiment 2. The square frames were similar to the LFs in Experiments 1 and 4, except that their inner contours were circular $\left(1.2^{\circ}\right.$ in diameter $)$. The circular frames were also $1.2^{\circ}$ in diameter. In both Experiments $6 \mathrm{~A}$ and $6 \mathrm{~B}$, there were an NF condition and two LF conditions (vertical and $30^{\circ}$-tilted square frames in $6 \mathrm{~A}$, circular frames and $45^{\circ}$-tilted square frames in $6 \mathrm{~B}$ ) in two search tasks: search for a vertical line among $30^{\circ}$-tilted lines and for a tilted line among vertical lines. Twelve new subjects ( 6 for each experiment, 3 females and 9 males, 23-33 years of age) from Tokyo Metropolitan University volun-

Table 5

Percentages of Errors in Experiment 5

\begin{tabular}{|c|c|c|c|c|c|c|c|c|c|}
\hline \multirow[b]{2}{*}{ Frame } & \multirow[b]{2}{*}{ Target } & \multicolumn{4}{|c|}{ Present Trial } & \multicolumn{4}{|c|}{ Absent Trial } \\
\hline & & 1 & 4 & 10 & 16 & 1 & 4 & 10 & 16 \\
\hline \multirow[t]{2}{*}{$\mathrm{NF}$} & Vertical & 1.4 & 3.5 & 9.7 & 9.7 & 0.7 & 2.1 & 1.4 & 0.0 \\
\hline & Tilted & 4.9 & 3.5 & 4.9 & 4.9 & 6.3 & 2.8 & 2.8 & 1.4 \\
\hline \multirow[t]{2}{*}{ LF vertical } & Vertical & 2.8 & 6.9 & 18.1 & 28.5 & 0.7 & 2.8 & 3.5 & 3.5 \\
\hline & Tilted & 2.8 & 3.5 & 9.7 & 16.0 & 2.8 & 6.3 & 1.4 & 4.2 \\
\hline \multirow[t]{2}{*}{ LF tilted } & Vertical & 2.8 & 2.1 & 12.5 & 15.3 & 1.4 & 3.5 & 4.2 & 6.9 \\
\hline & Tilted & 0.7 & 4.2 & 18.8 & 35.4 & 3.5 & 2.1 & 2.8 & 4.9 \\
\hline
\end{tabular}

Note-NF, no frame; LF, local frame. 
(A)

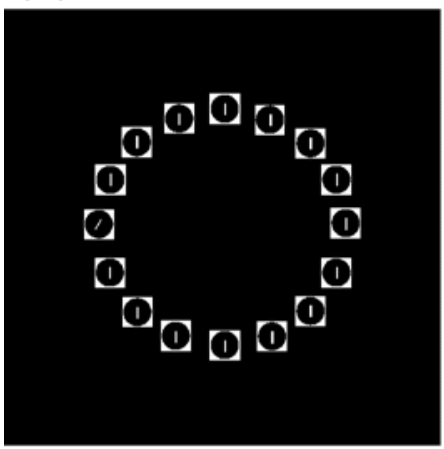

(C)

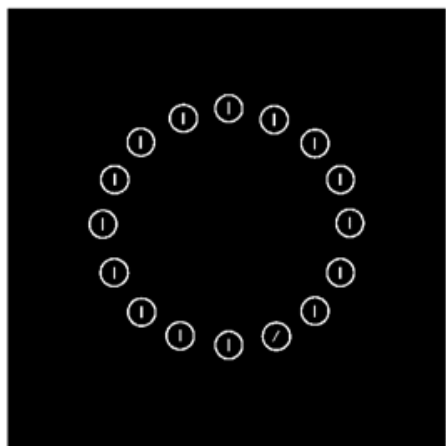

(B)

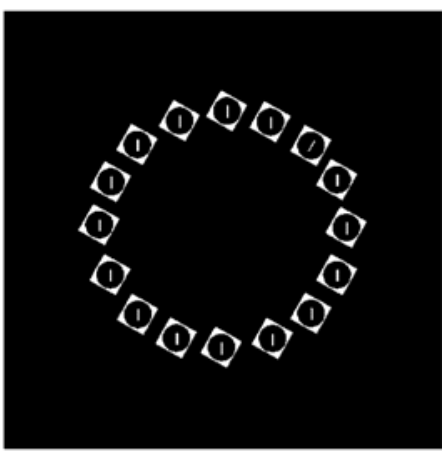

(D)

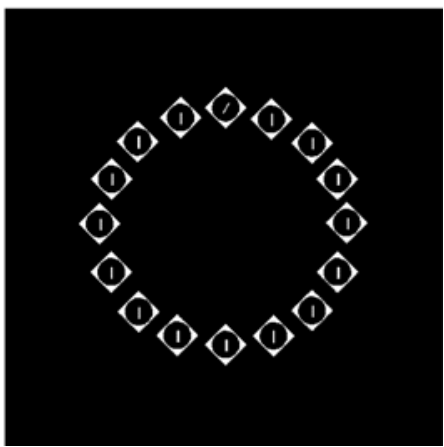

Figure 12. Examples of search displays in Experiments 6A and 6B. All the examples represent 16-item target-present trials in search for a tilted target with (A) vertical local frames (LFs), (B) $30^{\circ}$-tilted LFs, (C) circular LFs, and (D) $45^{\circ}$ tilted LFs.

teered for participation. All the subjects performed the two search tasks under the three frame conditions. In all other respects, the method was identical to that in Experiment 2.

\section{Results}

Experiment 6A. Figure 13 shows the mean correct RTs for the present and absent trials. The results were largely consistent with those in Experiment 5 . In the NF condition, an asymmetry was observed between the two search tasks. An ANOVA showed only a significant main effect of target type $[F(1,5)=14.87, p<.05]$. By close inspection of the data, the lack of an effect of set size $[F(3,15)=3.24]$ and of a target $\times$ set interaction $[F(3,15)=3.34]$ was due to intersubject variability in the RTs for the target-absent trials. When we confined analysis to RTs in the target-present trials, there were significant main effects of target type $[F(1,5)=45.05$, $p<.01]$ and set size $[F(3,15)=7.70, p<.05]$ and a significant interaction of target type and set size $[F(3,15)=$ $5.19, p<.05]$.

In comparison with the NF condition, both LFs produced significantly slower searches for the vertical target $[F(1,5)=28.01$ for the vertical LF and $F(1,5)=37.15$ for the tilted LF] and for the tilted target $[F(1,5)=20.30$ for the vertical LF and $F(1,5)=38.14$ for the tilted LF; all $p$ s $<.01]$. For the vertical LF, there was a clear asymmetry between the two search tasks, whereas there was no asymmetry for the tilted LF. A four-factor ANOVA showed, among other effects, significant main effects of frame orientation $[F(1,5)=13.63, p<.05]$ and set size $[F(3,15)=39.59, p<.01]$ and significant interactions of target type and frame orientation $[F(1,5)=12.14, p<$ $.05]$, frame orientation and set size $[F(3,15)=12.03$, $p<.01]$, and target type, frame orientation, and set size $[F(3,15)=7.21, p<.05]$. Subsequent ANOVAs showed that for the vertical LF, search for the vertical target was significantly slower $[F(1,5)=21.97, p<.01]$ and increased more rapidly with increases in set size $[F(3,15)=$ $8.72, p<.05]$ than search for the tilted target. For the tilted LF, there was no significant effect of target type $[F(1,5)=0.02]$ and no target type $\times$ set size interaction $[F(3,15)=0.07]$.

Table 6 presents the percentages of errors in this experiment. Similar to the results of Experiments 3 and 5, the error rates were higher when the target and the frame were matched in orientation than when they were not. The correlations between the mean RTs and the error rates were $R(22)=.874$ in the present trials and $R(22)=$ 

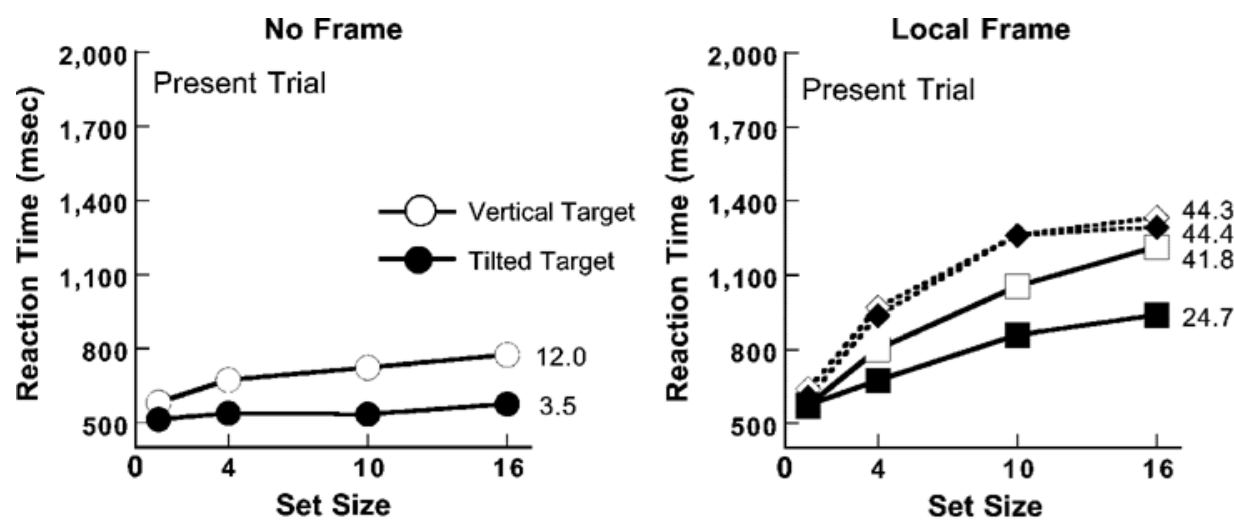

\section{Frame Vertical

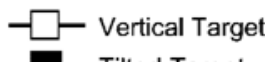

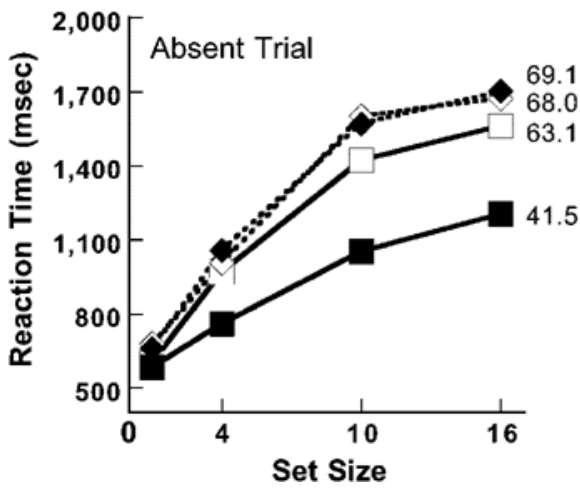

Frame Tilted

$-\diamond-.-$ Vertical Target

...- Tilted Target

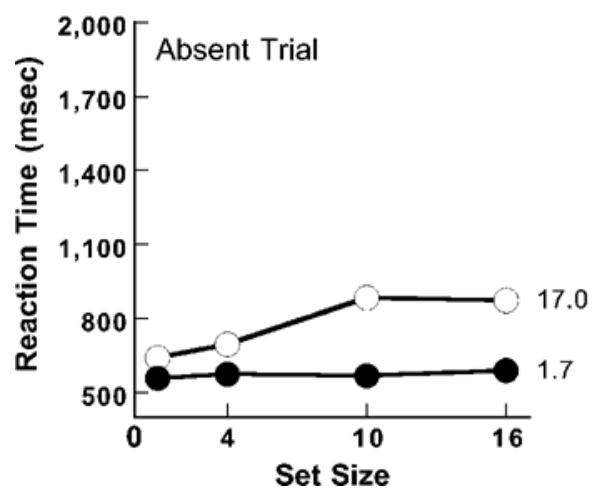

Figure 13. Mean correct reaction times (RTs) in Experiment 6A, together with the search rates (msec/item) for the RT functions.

.649 in the absent trials (both $p \mathrm{~s}<.01$ ). For the vertical target alone, $R(10)=.916, p<.01$, in the present trials, and $R(10)=.515$, n.s., in the absent trials. For the tilted target, $R(10)=.830$ in the present trials and $R(10)=.852$ in the absent trials (both $p$ s $<.01$ ). Therefore, the differences in RTs among the frame conditions may not have been contaminated by a speed-accuracy tradeoff.

Experiment 6B. Figure 14 shows the mean correct RTs for the present and absent trials. Again, there was an asymmetry between the two search tasks in the NF condition. An ANOVA showed significant main effects of target type $[F(1,5)=23.90, p<.01]$, trial type $[F(1,5)=22.94, p<.01]$, and set size $[F(3,15)=7.20$, $p<.05]$ and a significant interaction of target type and set size $[F(3,15)=8.51, p<.01]$.
In comparison with the NF condition, both circular and $45^{\circ}$ LFs produced significantly slower searches for the vertical target $[F(1,5)=38.53$ for the circular LF and $F(1,5)=98.74$ for the $\left.45^{\circ} \mathrm{LF}\right]$ and for the tilted target $[F(1,5)=46.31$ for the circular LF and $F(1,5)=281.23$ for the $45^{\circ} \mathrm{LF}$; all $\left.p \mathrm{~s}<.01\right]$. However, the patterns of results were quite different between the two types of frames. For the circular LF (inverted triangles), the asymmetry between the two search tasks was enlarged from that observed in the NF condition, whereas for the $45^{\circ} \mathrm{LF}$ (diamonds), there was no clear asymmetry in target-present trials. A four-factor ANOVA showed, among other effects, significant main effects of target type $[F(1,5)=$ $8.75, p<.05]$, frame type $[F(1,5)=10.73, p<.05]$, and set size $[F(3,15)=145.52, p<.01]$ and significant in-

Table 6

Percentages of Errors in Experiment 6A

\begin{tabular}{|c|c|c|c|c|c|c|c|c|c|}
\hline \multirow[b]{2}{*}{ Frame } & \multirow[b]{2}{*}{ Target } & \multicolumn{4}{|c|}{ Present Trial } & \multicolumn{4}{|c|}{ Absent Trial } \\
\hline & & 1 & 4 & 10 & 16 & 1 & 4 & 10 & 16 \\
\hline \multirow[t]{2}{*}{$\mathrm{NF}$} & Vertical & 1.4 & 3.5 & 4.2 & 9.7 & 4.2 & 3.5 & 4.9 & 1.4 \\
\hline & Tilted & 4.2 & 2.8 & 1.4 & 9.0 & 2.1 & 1.4 & 0.0 & 0.0 \\
\hline \multirow[t]{2}{*}{ LF vertical } & Vertical & 0.7 & 4.9 & 17.4 & 30.6 & 1.4 & 2.8 & 2.1 & 4.2 \\
\hline & Tilted & 2.1 & 4.2 & 7.6 & 11.8 & 1.4 & 0.0 & 0.7 & 2.8 \\
\hline \multirow[t]{2}{*}{ LF tilted } & Vertical & 2.8 & 9.0 & 16.7 & 23.6 & 2.1 & 2.1 & 3.5 & 17.4 \\
\hline & Tilted & 0.7 & 3.5 & 16.7 & 32.6 & 1.4 & 2.8 & 5.6 & 9.7 \\
\hline
\end{tabular}

Note-NF, no frame; LF, local frame. 

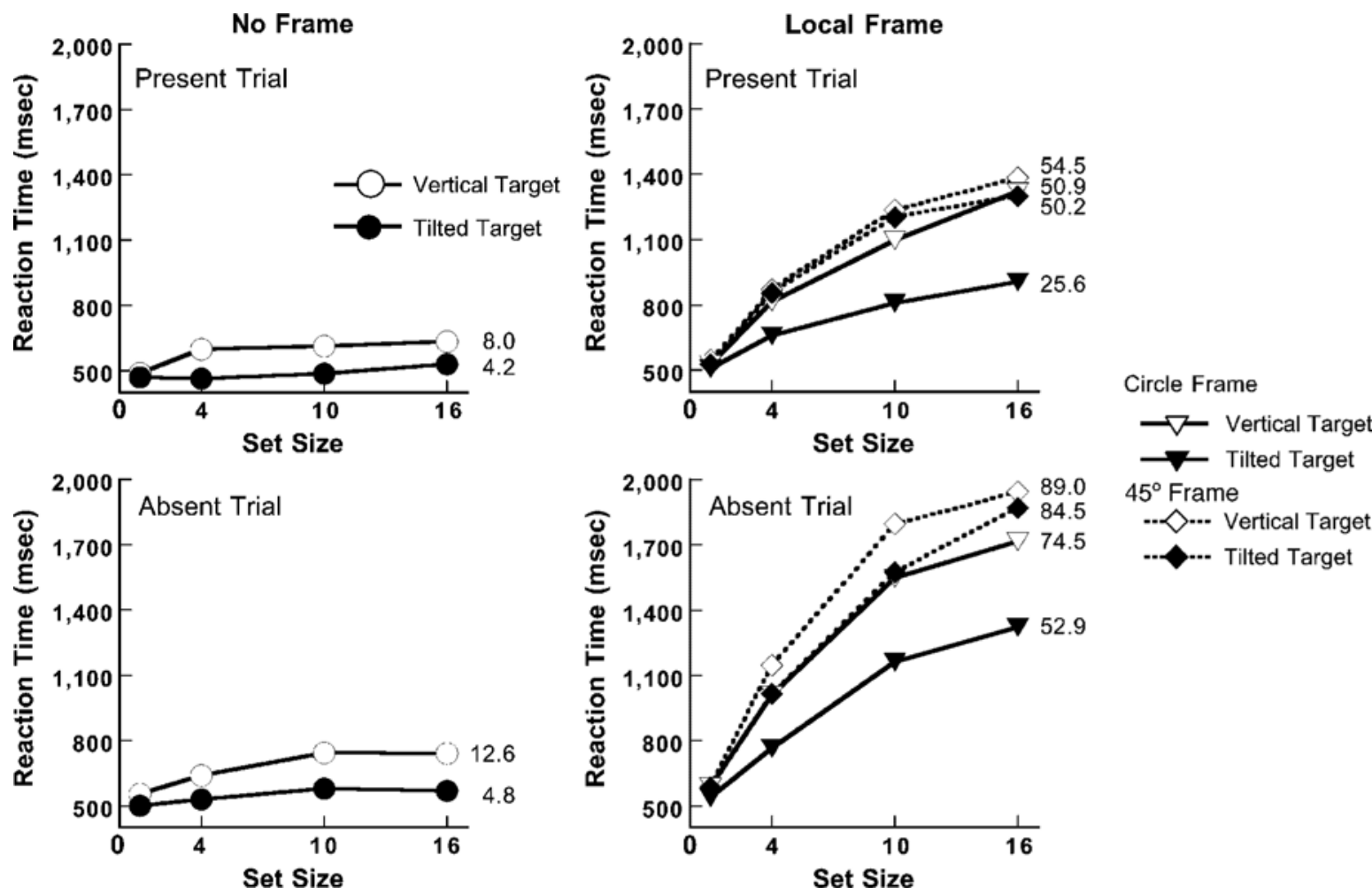

Figure 14. Mean correct reaction times (RTs) in Experiment 6B, together with the search rates (msec/item) for the RT functions.

teractions of target type and frame orientation $[F(1,5)=$ $14.95, p<.05]$, frame orientation and set size $[F(3,15)=$ $7.12, p<.05]$, and target type, frame orientation, and set size $[F(3,15)=7.80, p<.05]$. Subsequent ANOVAs showed that for the circular LF, the search for the vertical target was significantly slower $[F(1,5)=20.98, p<$ $.01]$ and increased more rapidly with increases in set size $[F(3,15)=15.66, p<.01]$ than the search for the tilted target. For the $45^{\circ} \mathrm{LF}$, there was no significant effect of target type $[F(1,5)=1.36]$ and no target type $\times$ set size interaction $[F(3,15)=1.09]$.

Table 7 presents the percentages of errors in this experiment. The correlations between the mean RTs and the error rates were $R(22)=.882, p<.01$, in the present trials, and $R(22)=-.332$, n.s., in the absent trials.
For the vertical target alone, $R(10)=.883, p<.01$, in the present trials, and $R(10)=-.294$, n.s., in the absent trials. For the tilted target, $R(10)=.902, p<.01$, in the present trials, and $R(10)=-.321$, n.s., in the absent trials. Therefore, the differences in RTs among the frame conditions may not have been contaminated by a speedaccuracy tradeoff.

\section{Discussion}

The results of Experiment $6 \mathrm{~A}$ replicated the main findings of Experiment 5 and confirmed that the interference effects of the LF vary with frame and target orientations. The results of Experiment 6B showed, unexpectedly, that the pattern of search performance was quite different between the circular and the $45^{\circ} \mathrm{LFs}$.

Table 7

Percentages of Errors in Experiment 6B

\begin{tabular}{|c|c|c|c|c|c|c|c|c|c|}
\hline \multirow[b]{2}{*}{ Frame } & \multirow[b]{2}{*}{ Target } & \multicolumn{4}{|c|}{ Present Trial } & \multicolumn{4}{|c|}{ Absent Trial } \\
\hline & & 1 & 4 & 10 & 16 & 1 & 4 & 10 & 16 \\
\hline \multirow[t]{2}{*}{$\mathrm{NF}$} & Vertical & 4.9 & 2.8 & 5.6 & 6.2 & 3.5 & 2.8 & 5.6 & 2.1 \\
\hline & Tilted & 3.5 & 2.8 & 4.9 & 4.9 & 5.6 & 2.1 & 2.8 & 2.1 \\
\hline \multirow[t]{2}{*}{ LF circular } & Vertical & 0.0 & 2.8 & 11.8 & 20.1 & 0.7 & 0.7 & 2.8 & 2.1 \\
\hline & Tilted & 1.4 & 6.3 & 4.2 & 11.8 & 4.2 & 2.1 & 4.2 & 4.2 \\
\hline \multirow[t]{2}{*}{ LF $45^{\circ}$-tilted } & Vertical & 1.4 & 4.2 & 10.4 & 16.0 & 1.4 & 4.9 & 1.4 & 0.0 \\
\hline & Tilted & 2.8 & 5.6 & 11.8 & 15.3 & 2.8 & 2.8 & 2.8 & 0.7 \\
\hline
\end{tabular}

Note-NF, no frame; LF, local frame. 
With the circular LFs, there was a marked asymmetry between search for a vertical target and that for a tilted target, owing to the much slowed performance for the vertical target, as compared with the NF condition. It is noteworthy that similar patterns of results were obtained for the vertical LF in the previous experiments - that is, Experiments 1, 2, 5, and 6A (see Figures 2, 4, 11, and 13). With the $45^{\circ} \mathrm{LFs}$, searches were slowed for both types of targets, but there was no asymmetry between them. This resembles the results obtained for the tilted LFs in Experiments 4, 5, and 6A (Figures 9, 11, and 13). We assumed that the $45^{\circ} \mathrm{LFs}$ would not be perceptually oriented, but the results indicate that they worked like other tilted LFs in search for line orientation. These results suggest a distinction between tilted LFs and nontilted (vertical and circular) LFs in terms of interference effects.

Although the present experiments do not allow us to specify the mechanisms underlying the two kinds of interference effects, the grouping process in visual search (see Bacon \& Egeth, 1991; Humphreys et al., 1989) provides a likely account for the effects of the nonvertical LFs. An important aspect of multi-item grouping is that search rates depend on the number of separate clumps into which the items are grouped (Grossberg, Mingolla, \& Ross, 1994). Other things being equal, a search will be faster as items present in the display are grouped into fewer clumps (in other words, more items are grouped into one clump). Given the use of identical distractor lines in the present study, grouping was easy when the items were presented with no frame, and they may have been grouped into only a few clumps, even for set sizes of 10 and 16. Adding LFs around the items would interfere with the grouping process, so that items would be grouped into a large number of small clumps (each of which would contain no more than a few items), resulting in relatively slow search rates. In this context, the increase in search rate from the NF condition would reflect the increase in number of clumps. If we assume that the size of interference, or the degree to which the number of clumps is increased from that in the NF condition, does not differ because of the orientation of the distractors (vertical or tilted) used, it would follow that the increase in search rate caused by adding LFs would be unchanged in search for a tilted among vertical lines and vice versa. This is what we found with the circular LFs (inverted triangles in Figure 14). Although the search rates obtained with the circular LF were quite different between the two search tasks (50.9 and $25.6 \mathrm{msec} /$ item for the vertical and the tilted targets, respectively, in the present trials), they were equally proportional to those in the NF condition $(50.9 / 8.0=6.4$ for the vertical and 25.6/4.2 $=6.1$ for the tilted target). By and large, the data for the vertical LFs showed similar tendencies (except for Experiment 6A): The ratios of search rates in the LF condition to those in the corresponding NF condition for the vertical and the tilted target were, respectively, 2.3 and 2.1 (Experiment 1), 2.0 and 1.7 (Experiment 2), 3.4 and 5.1 (Experiment 5), and 3.5 and 7.1 (Experiment 6A). By this account, the enlarged asymmetry observed for the circular and the vertical LFs was the result of a relative increase in the number of clumps by the same amount for the two search tasks.

To elaborate the above account more quantitatively, we will use the following equation, which models targetpresent RTs:

$$
\mathrm{RT}=R+1 / 2 \times K \times(N+1) .
$$

This is a slight modification of an equation proposed by Grossberg et al. (1994). $R$ corresponds to the time necessary to perform basic operations of visual search, such as retinotopic feature registration and motor response, $K$ is the combined time required to segment items into clumps and to select and identify a clump that contains a target, and $N$ is the mean number of clumps for each set size (for details, see Grossberg et al., 1994). In applying the equation to the mean RTs for the circular LF and the NF conditions in Experiment 6B (see Table 8), which was performed by the same group of subjects, we will assume that $R$ is constant for the two conditions, as well as for the two search tasks, whereas $K$ is different between the two tasks (but equal for the two conditions), because target identification would be faster in a search for a tilted line than in that for a vertical line (i.e., search asymmetry). We chose $R=410 \mathrm{msec}$ and $K=160 \mathrm{msec}$ (vertical target) and $100 \mathrm{msec}$ (tilted target), which modeled the data well. We also set $N$ to 0 (no grouping) for

Table 8

Mean Reaction Times (RTs) and Estimated Number of Grouped Regions $(N)$ for Target-Present Trials in Experiment 6B

\begin{tabular}{|c|c|c|c|c|c|c|c|c|}
\hline \multirow[b]{3}{*}{$\underline{\text { Set Size }}$} & \multicolumn{4}{|c|}{ Vertical Target } & \multicolumn{4}{|c|}{ Tilted Target } \\
\hline & \multicolumn{2}{|c|}{ Mean RT } & \multicolumn{2}{|c|}{$N$} & \multicolumn{2}{|c|}{ Mean RT } & \multicolumn{2}{|c|}{$N$} \\
\hline & NF & $\mathrm{CF}$ & NF & $\mathrm{CF}$ & $\mathrm{NF}$ & $\mathrm{CF}$ & NF & $\mathrm{CF}$ \\
\hline 1 & 487 & 525 & 0 & 0 & 468 & 506 & 0 & 0 \\
\hline 4 & 597 & 813 & 1.34 & 4.04 & 462 & 659 & 0.03 & 3.98 \\
\hline 10 & 612 & 1,096 & 1.53 & 7.58 & 485 & 807 & 0.50 & 6.94 \\
\hline 16 & 631 & 1,319 & 1.76 & 10.36 & 528 & 905 & 1.36 & 8.90 \\
\hline Slope & 8.0 & 50.9 & 0.10 & 0.66 & 4.2 & 25.6 & 0.09 & 0.56 \\
\hline Ratio & & 6.4 & & 6.6 & & 6.1 & & 6.2 \\
\hline
\end{tabular}

Note- $N$ was computed by fitting Equation 1 to the mean RTs, except for set size 1 where $N$ was set to 0 (no grouping). See the text for details. NF, no frame; CF, circular frame. 
set size 1 . Given those constraints, $N$ was estimated separately for set sizes 4,10 , and 16 for each task and frame condition. Table 8 shows the results, together with the rate at which the estimates of $N$ increased with set size (computed by linear regression). In either search task, $N$ was larger and increased more rapidly for the circular LF condition. The ratio of the increasing rates of $N$ for the LF to that for the NF condition closely matched the ratio of search rates.

Such an interference-in-grouping account is not readily applicable to the effects of the tilted LFs, which slowed search rates for the two tasks indistinguishably (i.e., no asymmetry). Since there was a clear asymmetry in the corresponding NF condition (except for Experiment 4), the search rate ratio of the LF to the NF condition was different between the two tasks: It was larger in search for a tilted target than in search for a vertical target. To accommodate the grouping account to this fact, we need to assume also that the size of interference (the degree to which the number of clumps was increased) by the tilted LF was larger for the vertical distractors (tilted target) than for the tilted distractors (vertical target) or simply that adding the tilted LFs caused the number of clumps to be equal for the two types of distractors, irrespective of the number of clumps when there was no frame. However, we have no theoretical or empirical grounds for either assumption. We have considered alternative explanations, including local orientation contrast (Nothdurft, 1993) and mechanisms other than interference (e.g., conjunction of target orientation and frame orientation; Bilsky \& Wolfe, 1995), but none seems to be satisfactory.

\section{GENERAL DISCUSSION}

A major finding in the present study is that adding a frame (LF) around each line item causes substantial changes in search efficiency for line orientation. The asymmetry between searches for a vertical line and those for a tilted line was reversed when the LF was tilted at the same angle as the tilted line (Experiments 1 and 2). This reversed asymmetry was also obtained with the tilted LRC, which was a variation of the LF made only of left and right component lines (Experiment 3 ). Other variations of the LF (top and bottom component lines, $15^{\circ}$ - and $45^{\circ}$-tilted angles, and gapped and circular contours) did not lead to a reversed asymmetry, but they greatly changed efficiency in the two search tasks.

We have identified three sources that contribute to the changes induced by LFs. First, left and right component lines serve as additional distractors when the LF and the target are of the same orientation. Second, top and bottom component lines induce orientation contrast to the inside line and distort the apparent orientation of the line. The LRC and TBC conditions in Experiment 2 provided strong evidence for these two sources. A third source, although shown less conclusively than the first two, is interference in orientation processing of adjacent lines (Hayward \& Burke, 2000). Supportive evidence for the interference effect comes from the results of Experiments 4, 5, 6A, and 6B, where the distraction and the orientation contrast effects had little effect. Together with the results of the other experiments, there appear to be two kinds of interference effects: one for nontilted (vertical and circular) LFs and the other for tilted LFs. Whereas the interference effects of nontilted LFs can be explained well in terms of multi-item grouping (Grossberg et al., 1994), we have as yet been unable to find a reasonable account for the effects of tilted LFs. Further research is needed to solve this problem.

In Experiment 1, we suggested that the reference standard for line orientation would also be implicated in the effects of LF. The reference standard hypothesis fit the results of Experiment 1, particularly the reversed asymmetry for the tilted LF. However, the subsequent experiments did not provide further support for the account. Experiment 3 showed that the tilted LRC, serving as an additional distractor, replicated the reversed asymmetry. In Experiment 4, the slow searches in both tasks with the $15^{\circ}$ - and $45^{\circ}$-tilted LFs contradicted the reference standard hypothesis. These results indicate that the LF, at least in the form used in the present study, will not shift the reference standard in the way suggested for the large frame (Treisman, 1985), or it will shift the reference standard, but its effect will be too weak to be evident, as compared with the effects of the other sources. In either case, shifting of the reference standard contributes little to the effects of the LF used in the present study.

So far in the present article, we have based our discussion on an assumption that an asymmetry for vertical and tilt orientations is inherent in human orientation processing, whatever the mechanism is (e.g., Marendaz, 1998; Treisman, 1985). Recently, Rosenholtz (2001) proposed a theoretical account for search asymmetries without reliance on the asymmetric nature of search mechanisms. She argued that the asymmetric performance reported in the literature was due, with few exceptions, to asymmetric experimental designs, not to asymmetric mechanisms. She demonstrated that several experiments showing asymmetric performance in search for motion and for color were asymmetric in design when the target and the distractors were represented in a proper feature space and when the background of the search display was taken into account. In the case of orientation, such a background was a frame surrounding the display, whether it was an experimentally induced one (Treisman, 1985) or the border of the presentation monitor, that made the experimental design asymmetric. As an alternative to asymmetric mechanisms, Rosenholtz proposed that search efficiency increases as a function of target saliency, which is represented in a feature space as the distance between the target and the distractors. To take the background effect into account, she assumed that the background serves as an additional distractor. Her account would apply well to asymmetry reversal by tilted LFs, as well as to that by a tilted frame surround- 
ing a search display (Treisman, 1985); the target would be less salient when the target and the frame (serving as a distractor) had the same orientation than when they were different in orientation. However, some of the other findings in the present study are not readily accounted for by her saliency model. In the case of a $45^{\circ}$-tilted frame, the model predicts that the search for a $30^{\circ}$-tilted target would be less efficient than the search for a vertical target, since the frame is more similar in orientation to the $30^{\circ}$ target, thus making it less salient, than to the vertical $\left(0^{\circ}\right)$ target. As we have shown, searches for a $30^{\circ}$ target and for a vertical target are equally inefficient with a $45^{\circ}$ frame (Experiments 4 and $6 \mathrm{~B}$ ). We have also shown clear search asymmetries for vertical and tilted targets when there is no experimentally induced frame or visible monitor frame (NF conditions in Experiments 2, 6A, and 6B). Therefore, with regard to the present study, whether a search asymmetry reflects asymmetric experimental designs or asymmetric search mechanisms remains an open question.

Finally, our original purpose in introducing the LF in the present study was to relate frame effects in visual search tasks to RF effects. In the present study, we have shown that the effects of LF are largely changed by manipulations of its physical properties - that is, tilt orientation, removal of component lines, and addition of gaps, which are known to modulate RF effects (Spinelli et al., 1991; Streibel et al., 1980; Wenderoth \& Beh, 1977). As was mentioned earlier, research into RF effects has provided clear evidence for orientation contrast effects between a frame contour and an inside line. In addition, our proposal that three different sources contribute to the effects of LF is akin to a recent view of RF effects suggesting three underlying mechanisms (Spinelli, Antonucci, Daini, \& Zoccolotti, 1995; Wenderoth \& Johnstone, 1988); one is the orientation contrast, and the others are visual-vestibular interactions and global figural processing. Visual-vestibular interactions cause a subjective body tilt in response to the viewing of a tilted frame (e.g., Sigman, Goodenough, \& Flanagan, 1978, 1979). Global figural processing provides one of the four symmetry axes of a square frame (one vertical, one horizontal, and two diagonal) as a judging standard for rod orientation (Beh et al., 1971; Wenderoth \& Beh, 1977). These three mechanisms are simultaneously at work in an RF display, with their relative contributions to the resulting RF effects being dependent on the experimental parameters in a given situation (Spinelli et al., 1995; Zoccolotti et al., 1992). It seems unlikely that visualvestibular interactions and global figural processing were involved in the effects of the LF observed in the present study. ${ }^{5}$ Visual-vestibular interactions do not operate very effectively with small frames such as the LFs in the present study (Zoccolotti et al., 1992). According to the global figural processing view, vertical and $45^{\circ}$-tilted LFs should yield similar, if not identical, search performance, because both frames possess identical symmetry axes, but we found that the two frames yielded quite dif- ferent results (Experiments 4 and 6B). Proper control of relevant parameters, such as an observer's posture and frame size, may reveal more precisely the relation between RF and LF effects.

\section{REFERENCES}

BACON, W. F., \& EGETH, H. E. (1991). Local processes in preattentive feature detection. Journal of Experimental Psychology: Human Perception \& Performance, 17, 77-90.

Beh, H. C., Wenderoth, P. M., \& Purcell, A. T. (1971). The angular function of a rod-and-frame illusion. Perception \& Psychophysics, 9 , 353-355.

Bilsky, A. B., \& Wolfe, J. M. (1995). Part-whole information is useful in visual search for size $\times$ size but not orientation $\times$ orientation conjunctions. Perception \& Psychophysics, 57, 749-760.

Carpenter, R. H. S., \& Blakemore, C. (1973). Interactions between orientations in human vision. Experimental Brain Research, 18, 287 303.

Carrasco, M., Mclean, T. L., Katz, S. M., \& Frieder, K. S. (1998). Feature asymmetries in visual search: Effects of display duration, target eccentricity, orientation and spatial frequency. Vision Research, 38, 347-374.

Coren, S., \& Hoy, V.S. (1986). An orientation illusion analog to the rod and frame: Relational effects in the magnitude of the distortion. Perception \& Psychophysics, 39, 159-163.

DOHERTY, L. M., \& Foster, D. H. (2001). Reference frame for rapid visual processing of line orientation. Spatial Vision, 14, 121-137.

DunCAN, J., \& Humphreys, G. W. (1989). Visual search and stimulus similarity. Psychological Review, 96, 433-458.

EBenholtZ, S. M. (1977). Determinants of the rod and frame effect: The role of retinal size. Perception \& Psychophysics, 22, 531-538.

Foster, D. H., \& WARD, P. A. (1991a). Asymmetries in oriented-line detection indicate two orthogonal filters in early vision. Proceedings of the Royal Society of London: Series B, 243, 75-81.

Foster, D. H., \& WARD, P. A. (1991b). Horizontal-vertical filters in early vision predict anomalous line-orientation frequencies. Proceedings of the Royal Society of London: Series B, 243, 83-86.

Grossberg, S., Mingolla, E., \& Ross, W. D. (1994). A neural theory of attentive visual search: Interactions of boundary, surface, spatial, and object representations. Psychological Review, 101, 470-489.

HaYward, W. G., \& BurKe, D. (2000, November). What pops out in pop-out? Paper presented at the 41 st Annual Meeting of the Psychonomic Society, New Orleans.

HE, Z. J., \& NAKAYAMA, K. (1992). Surface versus features in visual search. Nature, 359, 231-233.

Humphreys, G. W., Quinlan, P. T., \& RidDOCH, M. J. (1989). Grouping processes in visual search: Effects with single- and combinedfeature targets. Journal of Experimental Psychology: General, 118, 258-279.

MARENDAZ, C. (1998). Nature and dynamics of reference frames in visual search for orientation: Implications for early visual processing. Psychological Science, 9, 27-32.

Marendaz, C., Stivalet, P., Barraclough, L., \& Walkowiac, P. (1993). Effect of gravitational cues on visual search for orientation. Journal of Experimental Psychology: Human Perception \& Performance, 19, 1266-1277.

Meigen, T., LAgreZE, W.-D., \& BACH, M. (1994). Asymmetries in preattentive line detection. Vision Research, 34, 3103-3109.

MORI, S. (1997). Effects of absolute and relative gap sizes in visual search for closure. Canadian Journal of Experimental Psychology, 51, 112-124.

Mori, S., Kusano, T., \& Kataoka, A. (2000, November). Frame effects on visual search are not black-and-white. Paper presented at the 41st Annual Meeting of the Psychonomic Society, New Orleans. NothduRfT, H. C. (1991). Texture segmentation and pop-out from orientation contrast. Vision Research, 31, 1073-1078.

NothduRFT, H. C. (1992). Feature analysis and the role of similarity in preattentive vision. Perception \& Psychophysics, 52, 355-375.

NothduRfT, H. C. (1993). The role of features in preattentive vision: 
Comparison of orientation, motion and color cues. Vision Research, 33, 1937-1958.

Over, R., Broerse, J., \& Crassini, B. (1972). Orientation illusion and masking in central and peripheral vision. Journal of Experimental Psychology, 96, 25-31.

PASHler, H. (1987). Detecting conjunctions of color and form: Reassessing the serial search hypothesis. Perception \& Psychophysics, 41, 191-201.

Rock, I. (1990). The frame of reference. In I. Rock (Ed.), The legacy of Solomon Asch: Essays in cognition and social psychology (pp. 243268). Hillsdale, NJ: Erlbaum.

Rosenholtz, R. (2001). Search asymmetries? What search asymmetries? Perception \& Psychophysics, 63, 476-489.

Sigman, E., Goodenough, D. R., \& Flanagan, M. (1978). Subjective estimates of body tilt and the rod-and-frame test. Perceptual \& Motor Skills, 47, 1051-1056.

Sigman, E., Goodenough, D. R., \& Flanagan, M. (1979). Instructions, illusory self-tilt, and the rod-and-frame test. Quarterly Journal of Experimental Psychology, 31, 155-165.

Spinelli, D., Antonucci, G., DAini, R., \& Zoccolotti, P. (1995). Local and global visual mechanisms underlying individual differences in the rod-and-frame illusion. Perception \& Psychophysics, 57, 915-920.

Sinelli, D., Antonucci, G., Goodenough, D. R., Pizzamiglio, L., \& Zoccolotti, P. (1991). Psychophysiological mechanisms underlying the rod and frame illusion. In S. Wapner \& J. Demick (Eds.), Field dependence-independence: Cognitive style across the life span (pp. 37-60). Hillsdale, NJ: Erlbaum.

Streibel, M. J., Barnes, R. D., Julness, G. D., \& Ebenholtz, S. M. (1980). Determinants of the rod-and-frame effect: Role of organization and subjective contour. Perception \& Psychophysics, 27, 136140.

Treisman, A. (1985). Preattentive processing in vision. Computer Vision, Graphics, \& Image Processing, 31, 156-177.

TREISMAN, A. (1988). Features and objects: The 14th Bartlett memorial lecture. Quarterly Journal of Experimental Psychology, 40A, 201237.

TREISMAN, A., \& GORMICAN, S. (1988). Feature analysis in early vision: Evidence from search asymmetries. Psychological Review, 95, 1548.

Treisman, A., \& SATo, S. (1990). Conjunction search revisited. Journal of Experimental Psychology: Human Perception \& Performance, 16, 459-478.

Wenderoth, P. M. (1974). The distinction between the rod-and-frame illusion and the rod-and-frame test. Perception, 3, 205-212.

Wenderoth, P. [M.], \& BeH, H. (1977). Component analysis of orientation illusions. Perception, 6, 57-75.

Wenderoth,P. [M.], \& Johnstone, S. (1987). Possible neural substrates for orientation analysis and perception. Perception, 16, 693-709.

Wenderoth, P. [M.], \& JohnSTONE, S. (1988). The differential effects of brief exposures and surrounding contours on direct and indirect tilt illusions. Perception, 17, 165-176.
WITKIN, H. A., \& ASCH, S. E. (1948). Studies in space orientation: IV. Further experiments on perception of the upright with displaced visual fields. Journal of Experimental Psychology, 38, 762-782.

Wolfe, J. M. (1999). Visual search. In H. Pashler (Ed.), Attention (pp. 13-73). Hove, U.K.: Psychology Press.

Wolfe, J. M., Friedman-Hill, S. R., Stewart, M. I., \& O'Connell, K. M. (1992). The role of categorization in visual search for orientation. Journal of Experimental Psychology: Human Perception \& Performance, 18, 34-49.

Wolfe, J. M., O'Neill, P., \& BennetT, S. C. (1998). Why are there eccentricity effects in visual search? Visual and attentional hypotheses. Perception \& Psychophysics, 60, 140-156.

Zoccolotti,P., Antonucci, G., Goodenough, D. R., Pizzamiglio,L., \& SPINELLI, D. (1992). The role of frame size on vertical and horizontal observers in the rod-and-frame illusion. Acta Psychologica, 79, 171-187.

Zoccolotti, P., Antonucci, G., \& SPInelli, D. (1993). The gap between rod and frame influences the rod-and-frame effect with small and large inducing displays. Perception \& Psychophysics, 54, 14-19.

\section{NOTES}

1. Although we did not use formal methods to eliminate the jaggedness of the tilted lines (e.g., anti-aliasing technique), we adjusted the size of lines, as well as the viewing distance from the monitor, so that the jaggedness was not readily perceivable by the observer. None of the subjects in the present study reported that they had noticed the jaggedness of the lines or that such jaggedness had either helped or disturbed their performance during the experiment.

2. In this and subsequent ANOVAs reported in this article, the significance levels were computed using Huynh-Feldt corrected degrees of freedom for lack of sphericity in repeated measures.

3. This experiment and Experiments $6 \mathrm{~A}$ and $6 \mathrm{~B}$ were conducted after Experiments 1, 3, 4, and 5 had been completed.

4. The previous studies showed that changes in apparent orientation of line due to orientation contrast were less than $2^{\circ}$ (Over et al., 1972; Wenderoth \& Beh, 1977). One may argue that such small changes may not be sufficient to cause the substantial changes in search rates observed in the present study. We point out that in the studies of orientation contrast and RF effects, the observers were usually exposed to the stimuli for a long period of time when they set the rod, whereas exposure duration was limited in the present experiments until the subjects made a response. Wenderoth and Johnstone (1988) showed that the size of the tilt illusion due to orientation contrast increased as exposure duration was shortened. It is thus likely that the orientation contrast induced sufficiently large effects on the apparent orientation of the inside line to change search performance in the present experiment.

5. We thank Christian Marendaz for pointing this out.

(Manuscript received February 2, 2001; revision accepted for publication September 3, 2003.) 